\title{
Hyper-Kähler Hierarchies and their twistor theory
}

\author{
Maciej Dunajski, Lionel J. Mason \\ The Mathematical Institute, 24-29 St Giles, Oxford OX1 3LB, UK
}

\begin{abstract}
A twistor construction of the hierarchy associated with the hyper-Kähler equations on a metric (the anti-self-dual Einstein vacuum equations, ASDVE, in four dimensions) is given. The recursion operator $R$ is constructed and used to build an infinite-dimensional symmetry algebra and in particular higher flows for the hyper-Kähler equations. It is shown that $R$ acts on the twistor data by multiplication with a rational function. The structures are illustrated by the example of the Sparling-Tod (Eguchi-Hansen) solution.

An extended space-time $\mathcal{N}$ is constructed whose extra dimensions correspond to higher flows of the hierarchy. It is shown that $\mathcal{N}$ is a moduli space of rational curves with normal bundle $\mathcal{O}(n) \oplus \mathcal{O}(n)$ in twistor space and is canonically equipped with a Lax distribution for ASDVE hierarchies. The space $\mathcal{N}$ is shown to be foliated by four dimensional hyper-Kähler slices.

The Lagrangian, Hamiltonian and bi-Hamiltonian formulations of the ASDVE in the form of the heavenly equations are given. The symplectic form on the moduli space of solutions to heavenly equations is derived, and is shown to be compatible with the recursion operator.
\end{abstract}

\section{Introduction}

Roger Penrose's twistor theory gives rise to correspondences between solutions to differential equations on the one hand and unconstrained holomorphic geometry on the other. The two most prominent systems of nonlinear equations which admit such correspondences are the anti-self-dual vacuum Einstein equations (ASDVE) [23] which in Euclidean signature determine hyper-Kähler metrics, and the anti-self-dual YangMills equations (ASDYM) [31]. Richard Ward [32] observed that many lower-dimensional integrable systems are symmetry reductions of ASDYM. This has led to an overview of the theory of integrable systems [20], which provides a classification of those lower-dimensional integrable systems that arise as reductions of the ASDYM equations and a unification of the theory of such integrable equations as symmetry reduced versions of the corresponding theory of the ASDYM equations. In [20], Lagrangian and Hamiltonian frameworks for ASDYM were described together with a recursion operator. This leads to the corresponding structures for symmetry reductions of the ASDYM equations.

In this paper we investigate these structures for the second important system of equations - the ASDVE or hyper-Kähler equation (this system also admits known integrable systems as symmetry reductions [11]). We shall give a twistor-geometric construction of the hierarchies associated to the ASDVE in the 'heavenly' forms due to Plebański 25]. In this context it is more natural to work with complex (holomorphic) metrics on complexified space-times and so we use the term ASDVE equations rather than hyper-Kähler equations. Our considerations will generally be local in space-time which will be understood to be a region in $\mathbb{C}^{4}$.

In Section 2 we summarise the twistor correspondences for flat and curved spaces. We establish a spinor notation (which will not be essential for the subsequent sections) and recall basic facts about the ASD conformal condition and the geometry of the spin bundle. In Section 3 the recursion operator $R$ for the ASDVE is constructed as an integro- differential operator mapping solutions to the linearised heavenly equations to other solutions. We then use this to give an alternate development of the twistor correspondence by using $R$ to build a family of foliations by twistor surfaces. We show that $R$ corresponds to multiplication of the twistor data by a given twistor function. We then analyse the hidden symmetry algebra of the ASDVE, and use the recursion operator to construct Killing spinors. We illustrate the ideas using the example of the Sparling-Tod solution and show how $R$ can be used to construct rational curves with normal bundle $\mathcal{O}(1) \oplus \mathcal{O}(1)$ in the associated twistor space.

In Section 1 we give the twistor construction for the ASDVE hierarchies. The higher commuting flows can be thought of as coordinates on an extended space-time. This extended space-time has a twistor

*email: dunajski@maths.ox.ac.uk 
correspondence: it is the moduli space of rational curves with normal bundle $\mathcal{O}(n) \oplus \mathcal{O}(n)$ in a twistor space. This moduli space is canonically equipped with the Lax distribution for ASDVE hierarchies, and conversely that truncated hierarchies admit a Lax distribution that gives rise to such a twistor space. The Lax distribution can be interpreted as a connecting map in a long exact sequence of sheaves. In Section 5 we investigate the Lagrangian and Hamiltonian formulations of heavenly equations. The symplectic form on the moduli space of solutions to heavenly equations will be derived, and is shown to be compatible with the recursion operator.

We end this introduction with some bibliographical remarks. Significant progress towards understanding the symmetry structure of the heavenly equations was achieved by Boyer and Plebański [3, 4] who obtained an infinite number of conservation laws for the ASDVE equations and established some connections with the nonlinear graviton construction. Their results were later extended in papers of Strachan [26] and Takasaki [28, 29]. The present work is an extended version of [8, 9, 10.

\section{Preliminaries}

\subsection{Spinor notation}

We work in the holomorphic category with complexified space-times: thus space-time $\mathcal{M}$ is a complex four-manifold equipped with a holomorphic metric $g$ and compatible volume form $\nu$.

In four complex dimensions orthogonal transformations decompose into products of ASD and SD rotations

$$
S O(4, \mathbb{C})=(S L(2, \mathbb{C}) \times \widetilde{S L}(2, \mathbb{C})) / \mathbb{Z}_{2}
$$

The spinor calculus in four dimensions is based on this isomorphism. We use the conventions of Penrose and Rindler 24]. Indices will generally be assumed to be concrete unless stated otherwise: $a, b, \ldots$, $a=0,1 \ldots 3$ are four-dimensional space-time indices and $A, B, \ldots, A^{\prime}, B^{\prime}, \ldots, A=0,1$ etc. are twodimensional spinor indices. The tangent space at each point of $\mathcal{M}$ is isomorphic to a tensor product of the two spin spaces

$$
T^{a} \mathcal{M}=S^{A} \otimes S^{A^{\prime}}
$$

The complex Lorentz transformation $V^{a} \longrightarrow \Lambda_{b}^{a} V^{b}, \Lambda_{b}^{a} \Lambda_{d}^{c} g_{a c}=g_{b d}$, is equivalent to the composition of the SD and the ASD rotation

$$
V^{A A^{\prime}} \longrightarrow \lambda^{A}{ }_{B} V^{B B^{\prime}} \lambda^{A^{\prime}}{ }_{B^{\prime}}
$$

where $\lambda^{A}{ }_{B}$ and $\lambda^{A^{\prime}} B^{\prime}$ are elements of $S L(2, \mathbb{C})$ and $\widetilde{S L}(2, \mathbb{C})$.

Spin dyads $\left(o^{A}, \iota^{A}\right)$ and $\left(o^{A^{\prime}}, \iota^{A^{\prime}}\right)$ span $S^{A}$ and $S^{A^{\prime}}$ respectively. The spin spaces $S^{A}$ and $S^{A^{\prime}}$ are equipped with symplectic forms $\varepsilon_{A B}$ and $\varepsilon_{A^{\prime} B^{\prime}}$ such that $\varepsilon_{01}=\varepsilon_{0^{\prime} 1^{\prime}}=1$. These anti-symmetric objects are used to raise and lower the spinor indices. We shall use normalised spin frames so that

$$
o^{B} \iota^{C}-\iota^{B} o^{C}=\varepsilon^{B C}, \quad o^{B^{\prime}} \iota^{C^{\prime}}-\iota^{B^{\prime}} o^{C^{\prime}}=\varepsilon^{B^{\prime} C^{\prime}} .
$$

Let $e^{A A^{\prime}}$ be a null tetrad of 1 -forms on $\mathcal{M}$ and let $\nabla_{A A^{\prime}}$ be the frame of dual vector fields. The orientation is given by fixing the volume form

$$
\nu=e^{01^{\prime}} \wedge e^{10^{\prime}} \wedge e^{11^{\prime}} \wedge e^{00^{\prime}}
$$

Apart from orientability, $\mathcal{M}$ must satisfy some other topological restrictions for the global spinor fields to exist. We shall not take them into account as we work locally in $\mathcal{M}$.

The local basis $\Sigma^{A B}$ and $\Sigma^{A^{\prime} B^{\prime}}$ of spaces of ASD and SD two-forms are defined by

$$
e^{A A^{\prime}} \wedge e^{B B^{\prime}}=\varepsilon^{A B} \Sigma^{A^{\prime} B^{\prime}}+\varepsilon^{A^{\prime} B^{\prime}} \Sigma^{A B} .
$$

The first Cartan structure equations are

$$
\mathrm{d} e^{A A^{\prime}}=e^{B A^{\prime}} \wedge \Gamma_{B}^{A}+e^{A B^{\prime}} \wedge \Gamma_{B^{\prime}}^{A^{\prime}}
$$

where $\Gamma_{A B}$ and $\Gamma_{A^{\prime} B^{\prime}}$ are the $S L(2, \mathbb{C})$ and $\widetilde{S L}(2, \mathbb{C})$ spin connection one-forms. They are symmetric in their indices, and

$$
\Gamma_{A B}=\Gamma_{C C^{\prime} A B} e^{C C^{\prime}}, \quad \Gamma_{A^{\prime} B^{\prime}}=\Gamma_{C C^{\prime} A^{\prime} B^{\prime}} e^{C C^{\prime}}, \quad \Gamma_{C C^{\prime} A^{\prime} B^{\prime}}=o_{A^{\prime}} \nabla_{C C^{\prime}} \iota_{B^{\prime}}-\iota_{A^{\prime}} \nabla_{C C^{\prime} o_{B^{\prime}}}
$$

The curvature of the spin connection

$$
R_{B}^{A}=\mathrm{d} \Gamma_{B}^{A}+\Gamma_{C}^{A} \wedge \Gamma_{B}^{C}
$$


decomposes as

$$
R_{B}^{A}=C_{B C D}^{A} \Sigma^{C D}+(1 / 12) R \Sigma^{A}{ }_{B}+\Phi^{A}{ }_{B C^{\prime} D^{\prime}} \Sigma^{C^{\prime} D^{\prime}},
$$

and similarly for $R^{A^{\prime}}{ }_{B^{\prime}}$. Here $R$ is the Ricci scalar, $\Phi_{A B A^{\prime} B^{\prime}}$ is the trace-free part of the Ricci tensor $R_{a b}$, and $C_{A B C D}$ is the ASD part of the Weyl tensor

$$
C_{a b c d}=\varepsilon_{A^{\prime} B^{\prime}} \varepsilon_{C^{\prime} D^{\prime}} C_{A B C D}+\varepsilon_{A B} \varepsilon_{C D} C_{A^{\prime} B^{\prime} C^{\prime} D^{\prime}} .
$$

\subsection{The flat twistor correspondence}

The flat twistor correspondence is a correspondence between points in complexified Minkowski space, $\mathbb{C}^{4}$ (or its conformal compactification) and holomorphic lines in $\mathbb{C P}^{3}$.

The flat twistor correspondence has an invariant formulation in terms of spinors. A point in $\mathbb{C}^{4}$ has position vector with coordinates $(w, z, x, y)$. The isomorphism (2.2) is realised by

$$
x^{A A^{\prime}}:=\left(\begin{array}{cc}
y & w \\
-x & z
\end{array}\right), \quad \text { so that } g=\varepsilon_{A B \varepsilon_{A^{\prime} B^{\prime}}} \mathrm{d} x^{A A^{\prime}} \mathrm{d} x^{B B^{\prime}} .
$$

A two-plane in $\mathbb{C}^{4}$ is null if $g(X, Y)=0$ for every pair $(X, Y)$ of vectors tangent to it. The null planes can be self-dual (SD) or anti self-dual (ASD), depending on whether the tangent bi-vector $X \wedge Y$ is SD or ASD. The SD null planes are called $\alpha$-planes. The $\alpha$-planes passing through a point in $\mathbb{C}^{4}$ are parametrised by $\lambda=\pi_{0^{\prime}} / \pi_{1^{\prime}} \in \mathbb{C P}^{1}$. Tangents to $\alpha$-planes are spanned by two vectors

$$
L_{A}=\pi^{A^{\prime}} \frac{\partial}{\partial x^{A A^{\prime}}}
$$

which form the kernel of $\pi_{A^{\prime}} \pi_{B^{\prime}} \Sigma^{A^{\prime} B^{\prime}}$ The set of all $\alpha$-planes is called a projective twistor space and denoted $\mathcal{P} \mathcal{T}$. For $\mathbb{C}^{4}$ it is a three-dimensional complex manifold bi-holomorphic to $\mathbb{C P}^{3}-\mathbb{C P}^{1}$.

The five complex dimensional correspondence space $\mathcal{F}:=\mathbb{C}^{4} \times \mathbb{C P}^{1}$ fibres over $\mathbb{C}^{4}$ by $\left(x^{A A^{\prime}}, \lambda\right) \rightarrow x^{A A^{\prime}}$ and over $\mathcal{P} \mathcal{T}$ with fibres spanned by $L_{A}$. Twistor functions (functions on $\mathcal{P} \mathcal{T}$ ) pull back to functions on $\mathcal{F}$ which are constant on $\alpha$-planes, or equivalently satisfy $L_{A} f=0$.

Twistor space can be covered by two coordinate patches $U$ and $\widetilde{U}$, where $U$ is a complement of $\lambda=\infty$ and $\widetilde{U}$ is a compliment of $\lambda=0$. If $\left(\mu^{0}, \mu^{1}, \lambda\right)$ are coordinates on $U$ and $\left(\tilde{\mu}^{0}, \tilde{\mu}^{1}, \tilde{\lambda}\right)$ are coordinates on $\widetilde{U}$ then on the overlap

$$
\tilde{\mu}^{0}=\mu^{0} / \lambda, \quad \tilde{\mu}^{1}=\mu^{1} / \lambda, \quad \tilde{\lambda}=1 / \lambda .
$$

The local coordinates $\left(\mu^{0}, \mu^{1}, \lambda\right)$ on $\mathcal{P} \mathcal{T}$ pulled back to $\mathcal{F}$ are

$$
\mu^{0}=w+\lambda y, \quad \mu^{1}=z-\lambda x, \quad \lambda .
$$

We can introduce homogeneous coordinates on the twistor space

$$
\left(\omega^{A}, \pi_{A^{\prime}}\right)=\left(\omega^{0}, \omega^{1}, \pi_{0^{\prime}}, \pi_{1^{\prime}}\right):=\left(\mu^{0} \pi_{1^{\prime}}, \mu^{1} \pi_{1^{\prime}}, \lambda \pi_{1^{\prime}}, \pi_{1^{\prime}}\right) .
$$

The point $x^{A A^{\prime}} \in \mathbb{C}^{4}$ lies on the $\alpha$-plane corresponding to the twistor $\left(\omega^{A}, \pi_{A^{\prime}}\right) \in \mathcal{P} \mathcal{T}$ iff

$$
\omega^{A}=x^{A A^{\prime}} \pi_{A^{\prime}} .
$$

For $\pi_{A^{\prime}} \neq 0$ and $\left(\omega^{A}, \pi_{A^{\prime}}\right)$ fixed, The solution to $(2.6)$ is a complex two plane with tangent vectors of the form $\pi^{A^{\prime}} \nu^{A}$ for all $\nu^{A}$. Alternatively, if we fix $x^{A A^{\prime}}$, then (2.6) defines a rational curve, $\mathbb{C} \mathbb{P}^{1}$, in $\mathcal{P} \mathcal{T}$ with normal bundle $\mathcal{O}(1) \oplus \mathcal{O}(1)$.1 Kodaira theory guarantees that the family of such rational curves in $\mathcal{P} \mathcal{T}$ is four complex dimensional. There is a canonical (quadratic) conformal structure $\mathrm{d} s^{2}$ on $\mathbb{C}^{4}$ : the points $p$ and $q$ are null separated with respect to $\mathrm{d} s^{2}$ in $\mathbb{C}^{4}$ iff the corresponding rational curves $l_{p}$ and $l_{q}$ intersect in $\mathcal{P} \mathcal{T}$ at one point.

\subsection{Curved twistor spaces and the geometry of the primed spin bundle.}

Given a complex four-dimensional manifold $\mathcal{M}$ with curved metric $g$, a twistor in $\mathcal{M}$ is an $\alpha$-surface, i.e. a null two-dimensional surface whose tangent space at each point is an $\alpha$ plane. There are Frobenius integrability conditions for the existence of such $\alpha$-surfaces through each $\alpha$-plane element at each point and these are equivalent, after some calculation, to the vanishing of the self-dual part of the Weyl curvature, $C_{A^{\prime} B^{\prime} C^{\prime} D^{\prime}}$. Thus, given $C_{A^{\prime} B^{\prime} C^{\prime} D^{\prime}}=0$, we can define a twistor space $\mathcal{P} \mathcal{T}$ to be the three complex dimensional manifold of $\alpha$-surfaces in $\mathcal{M}$. If $g$ is also Ricci flat then $\mathcal{P} \mathcal{T}$ has further structures which are listed in the Nonlinear Graviton Theorem:

\footnotetext{
${ }^{1}$ Here $\mathcal{O}(n)$ denotes the line bundle over $\mathbb{C P}^{1}$ with transition functions $\lambda^{-n}$ from the set $\lambda \neq \infty$ to $\lambda \neq 0$ (i.e. Chern class $n)$.
} 
Theorem 2.1 (Penrose [23]) There is a 1-1 correspondence between complex ASD vacuum metrics on complex four-manifolds and three dimensional complex manifolds $\mathcal{P} \mathcal{T}$ such that

- There exists a holomorphic projection $\mu: \mathcal{P T} \longrightarrow \mathbb{C P}^{1}$

- $\mathcal{P} \mathcal{T}$ is equipped with a four complex parameter family of sections of $\mu$ each with a normal bundle $\mathcal{O}(1) \oplus \mathcal{O}(1)$, (this will follow from the existence of one such curve by Kodaira theory),

- Each fibre of $\mu$ has a symplectic structure $\Sigma_{\lambda} \in \Gamma\left(\Lambda^{2}\left(\mu^{-1}(\lambda)\right) \otimes \mathcal{O}(2)\right)$, where $\lambda \in \mathbb{C P}^{1}$.

To obtain real metrics on a real 4-manifold, we can require further that the twistor space admit an anti-holomorphic involution.

The correspondence space $\mathcal{F}=\mathcal{M} \times \mathbb{C P}^{1}$ is coordinatized by $(x, \lambda)$, where $x$ denotes the coordinates on $\mathcal{M}$ and $\lambda$ is the coordinate on $\mathbb{C P}^{1}$ that parametrises the $\alpha$-surfaces through $x$ in $\mathcal{M}$. We represent $\mathcal{F}$ as the quotient of the primed-spin bundle $S^{A^{\prime}}$ with fibre coordinates $\pi_{A^{\prime}}$ by the Euler vector field $\Upsilon=\pi^{A^{\prime}} / \partial \pi^{A^{\prime}}$. We relate the fibre coordinates to $\lambda$ by $\lambda=\pi_{0^{\prime}} / \pi_{1^{\prime}}$. A form with values in the line bundle $\mathcal{O}(n)$ on $\mathcal{F}$ can be represented by a homogeneous form $\alpha$ on the non-projective spin bundle satisfying

$$
\Upsilon\lrcorner \alpha=0, \quad \mathcal{L}_{\Upsilon} \alpha=n \alpha .
$$

The space $\mathcal{F}$ possesses a natural two dimensional distribution called the twistor distribution, or Lax pair, to emphasise the analogy with integrable systems. The Lax pair on $\mathcal{F}$ arises as the image under the projection $T S^{A^{\prime}} \longrightarrow T \mathcal{F}$ of the distribution spanned by

$$
\pi^{A^{\prime}} \partial_{A A^{\prime}}+\Gamma_{A A^{\prime} B^{\prime} C^{\prime}} \pi^{A^{\prime}} \pi^{B^{\prime}} \frac{\partial}{\partial \pi_{C^{\prime}}}
$$

on $T S^{A^{\prime}}$ where the $\partial_{A A^{\prime}}$ are a null tetrad for the metric on $\mathcal{M}$, and $\Gamma_{A A^{\prime} B^{\prime} C^{\prime}}$ are the components of the spin connection in the associated spin frame $\left(\partial_{A A^{\prime}}+\Gamma_{A A^{\prime} B^{\prime} C^{\prime}} \pi^{B^{\prime}} \frac{\partial}{\partial \pi_{C^{\prime}}}\right.$ is the horizontal distribution on $\left.S_{A^{\prime}}\right)$. We can also represent the Lax pair on the projective spin bundle by 1

$$
L_{A}=\left(\pi_{1^{\prime}}^{-1}\right)\left(\pi^{A^{\prime}} \partial_{A A^{\prime}}+f_{A} \partial_{\lambda}\right), \quad \text { where } f_{A}=\left(\pi_{1^{\prime}}^{-2}\right) \Gamma_{A A^{\prime} B^{\prime} C^{\prime}} \pi^{A^{\prime}} \pi^{B^{\prime}} \pi^{C^{\prime}} .
$$

The integrability of the twistor distribution is equivalent to $C_{A^{\prime} B^{\prime} C^{\prime} D^{\prime}}=0$, the vanishing of the self-dual Weyl spinor. When the Ricci tensor vanishes also, a covariant constant primed spin frame can be found so that $\Gamma_{A A^{\prime} B^{\prime} C^{\prime}}=0$. We assume this from now on.

The projective twistor space $\mathcal{P} \mathcal{T}$ arises as a quotient of $\mathcal{F}$ by the twistor distribution. With the Ricci flat condition, the coordinate $\lambda$ descends to twistor space and $\pi_{A^{\prime}}$ descends to the non-projective twistor space. It can be covered by two sets, $U=\{|\lambda|<1+\epsilon\}$ and $\tilde{U}=\{|\lambda|>1-\epsilon\}$. On the non-projective space we can introduce extra coordinates $\omega^{A}$ of homogeneity degree one so that $\left(\omega^{A}, \pi_{A^{\prime}}\right), \pi_{A^{\prime}} \neq \iota_{A^{\prime}}$ are homogeneous coordinates on $U$ and similarly $\left.\left(\tilde{\omega}^{A}, \pi_{A^{\prime}}\right), \pi_{A^{\prime}} \neq o_{A^{\prime}}\right)$ on $\tilde{U}$. The twistor space $\mathcal{P} \mathcal{T}$ is then determined by the transition function $\tilde{\omega}^{B}=\tilde{\omega}^{B}\left(\omega^{A}, \pi_{A^{\prime}}\right)$ on $U \cap \tilde{U}$.

The correspondence space has the alternate definition

$$
\mathcal{F}=\mathcal{P} \mathcal{T} \times\left.\mathcal{M}\right|_{Z \in l_{x}}=\mathcal{M} \times \mathbb{C P}^{1}
$$

where $l_{x}$ is the line in $\mathcal{P} \mathcal{T}$ that corresponds to $x \in \mathcal{M}$ and $Z \in \mathcal{P} \mathcal{T}$ lies on $l_{x}$. This leads to a double fibration

$$
\mathcal{M} \stackrel{p}{\longleftarrow} \mathcal{F} \stackrel{q}{\longrightarrow} \mathcal{P} \mathcal{T} .
$$

The existence of $L_{A}$ can also be deduced directly from the correspondence. From [23], points in $\mathcal{M}$ correspond to rational curves in $\mathcal{P} \mathcal{T}$ with normal bundle $\mathcal{O}^{A}(1):=\mathcal{O}(1) \oplus \mathcal{O}(1)$. The normal bundle to $l_{x}$ consists of vectors tangent to $x$ (horizontally lifted to $T_{(x, \lambda)} \mathcal{F}$ ) modulo the twistor distribution. Therefore we have a sequence of sheaves over $\mathbb{C P}^{1}$

$$
0 \longrightarrow D \longrightarrow \mathbb{C}^{4} \longrightarrow \mathcal{O}^{A}(1) \longrightarrow 0
$$

The map $\mathbb{C}^{4} \longrightarrow \mathcal{O}^{A}(1)$ is given by $V^{A A^{\prime}} \longrightarrow V^{A A^{\prime}} \pi_{A^{\prime}}$. Its kernel consists of vectors of the form $\pi^{A^{\prime}} \lambda^{A}$ with $\lambda^{A}$ varying. The twistor distribution is therefore $D=O(-1) \otimes S^{A}$ and so there is a canonical $L_{A} \in \Gamma\left(D \otimes \mathcal{O}(1) \otimes S_{A}\right)$, as given in (2.8).

${ }^{2}$ Various powers of $\pi_{1^{\prime}}$ in formulae like (2.8) guarantee the correct homogeneity. We usually shall omit them when working on the projective spin bundle. In a projection $S^{A^{\prime}} \longrightarrow \mathcal{F}$ we shall use the replacement formula

$$
\frac{\partial}{\partial \pi^{A^{\prime}}} \longrightarrow \frac{\pi_{A^{\prime}}}{\pi_{1^{\prime}}^{2}} \partial_{\lambda}
$$

This is because (on functions of $\lambda$ )

$$
\frac{\partial}{\partial \pi_{A^{\prime}}}\left(\frac{\pi_{0^{\prime}}}{\pi_{1^{\prime}}}\right)=\frac{\pi_{1^{\prime}} o^{A^{\prime}}-\pi_{0^{\prime}} A^{A^{\prime}}}{\pi_{1^{\prime}}{ }^{2}}=\frac{\pi^{A^{\prime}}}{\pi_{1^{\prime}}{ }^{2}} .
$$




\subsection{Some formulations of the ASD vacuum condition}

The ASD vacuum conditions $C_{A^{\prime} B^{\prime} C^{\prime} D^{\prime}}=0, \Phi_{A B A^{\prime} B^{\prime}}=0=R$ imply the existence of a normalised, covariantly constant frame $\left(o^{A^{\prime}}, \iota^{A^{\prime}}\right)$ of $S^{A^{\prime}}$, so that $\Gamma_{A A^{\prime} B^{\prime} C^{\prime}}=0$. One can further choose an unprimed spin frame so that the Lax pair (2.8) consists of volume-preserving vector fields on $\mathcal{M}$ :

Proposition 2.2 (Mason \& Newman [18].) Let $\widehat{\nabla}_{A A^{\prime}}=\left(\widehat{\nabla}_{00^{\prime}}, \widehat{\nabla}_{01^{\prime}}, \widehat{\nabla}_{10^{\prime}} \widehat{\nabla}_{11^{\prime}}\right)$ be four independent holomorphic vector fields on a four-dimensional complex manifold $\mathcal{M}$ and let $\nu$ be a nonzero holomorphic four-form. Put

$$
L_{0}=\widehat{\nabla}_{00^{\prime}}-\lambda \widehat{\nabla}_{01^{\prime}}, \quad L_{1}=\widehat{\nabla}_{10^{\prime}}-\lambda \widehat{\nabla}_{11^{\prime}} .
$$

Suppose that for every $\lambda \in \mathbb{C P}^{1}$

$$
\left[L_{0}, L_{1}\right]=0, \quad \mathcal{L}_{L_{A}} \nu=0 .
$$

Here $\mathcal{L}_{V}$ denotes the Lie derivative. Then

$$
\partial_{A A^{\prime}}=f^{-1} \widehat{\nabla}_{A A^{\prime}}, \quad \text { where } \quad f^{2}:=\nu\left(\widehat{\nabla}_{00^{\prime}}, \widehat{\nabla}_{01^{\prime}} \widehat{\nabla}_{10^{\prime}} \widehat{\nabla}_{11^{\prime}}\right),
$$

is a null-tetrad for an ASD vacuum metric. Every such metric locally arises in this way.

In [7] the last proposition is generalised to the hyper-Hermitian case. A choice of unprimed spin frame with $f^{2}=1$ is always possible and we shall assume this here-on so that $\nabla_{A A^{\prime}}=\widehat{\nabla}_{A A^{\prime}}$. For easy reference we rewrite the field equations (2.11) in full

$$
\begin{gathered}
{\left[\nabla_{A 0^{\prime}}, \nabla_{B 0^{\prime}}\right]=0,} \\
{\left[\nabla_{A 0^{\prime}}, \nabla_{B 1^{\prime}}\right]+\left[\nabla_{A 1^{\prime}}, \nabla_{B 0^{\prime}}\right]=0,} \\
{\left[\nabla_{A 1^{\prime}}, \nabla_{B 1^{\prime}}\right]=0 .}
\end{gathered}
$$

Let $\Sigma^{A^{\prime} B^{\prime}}$ be the usual basis of SD two-forms. On the correspondence space, define

$$
\Sigma(\lambda):=\Sigma^{A^{\prime} B^{\prime}} \pi_{A^{\prime}} \pi_{B^{\prime}}
$$

The formulation of the ASDVE condition dual to (2.11) is:

Proposition 2.3 (Plebański [25], Gindikin 12]) If a two-form of the form

$$
\Sigma(\lambda):=\Sigma^{A^{\prime} B^{\prime}} \pi_{A^{\prime}} \pi_{B^{\prime}}
$$

on the correspondence space satisfies

$$
\mathrm{d}_{h} \Sigma(\lambda)=0, \quad \Sigma(\lambda) \wedge \Sigma(\lambda)=0
$$

where $\mathrm{d}_{h}$ is the exterior derivative holding $\pi_{A^{\prime}}$ constant, then there exist one-forms $e^{A A^{\prime}}$ related to $\Sigma^{A^{\prime} B^{\prime}}$ by equation (2.5) which give an ASD vacuum tetrad.

Note that the simplicity condition in (2.16) arises from the condition that $\Sigma^{A^{\prime} B^{\prime}}$ comes from a tetrad.

To construct Gindikin's two-form starting from the twistor space, one can pull back the fibrewise complex symplectic structure on $\mathcal{P} \mathcal{T} \longrightarrow \mathbb{C P}^{1}$ to the projective spin bundle and fix the ambiguity by requiring that it annihilates vectors tangent to the fibres. The resulting two-form is $\mathcal{O}(2)$ valued. (To obtain Gindikin's two-form one should divide it by a constant section of $\mathcal{O}(2)$.)

Put $\Sigma^{0^{\prime} 0^{\prime}}=-\tilde{\alpha}, \Sigma^{0^{\prime} 1^{\prime}}=\omega, \Sigma^{1^{\prime} 1^{\prime}}=\alpha$. The second equation in (2.16) becomes

$$
\omega \wedge \omega=2 \alpha \wedge \tilde{\alpha}:=-2 \nu, \quad \alpha \wedge \omega=\tilde{\alpha} \wedge \omega=\alpha \wedge \alpha=\tilde{\alpha} \wedge \tilde{\alpha}=0 .
$$

Equations $(2.16)$ can be seen to arise from (2.11) by observing that $\Sigma(\lambda)$ can be defined by

$$
\varepsilon_{A B} \Sigma(\lambda)=\nu\left(L_{A}, L_{B}, \ldots, \ldots\right) .
$$

Note also that $L_{A}$ spans a two-dimensional distribution annihilating $\Sigma(\lambda)$.

The two one-forms $e^{A}:=\pi_{A^{\prime}} e^{A A^{\prime}}$ by definition annihilate the twistor distribution. Define $(1,1)$ tensors $\partial_{A^{\prime}}^{B^{\prime}}:=e^{A B^{\prime}} \otimes \nabla_{A A^{\prime}}$ so that

$$
e^{A} \otimes L_{A}=\pi_{B^{\prime}} \pi^{A^{\prime}} \partial_{A^{\prime}}^{B^{\prime}}=\partial_{0}+\lambda(\partial-\tilde{\partial})-\lambda^{2} \partial_{2}
$$

where $\left(\partial_{0^{\prime}}^{0^{\prime}}, \partial_{1^{\prime}}^{0^{\prime}}, \partial_{0^{\prime}}^{1^{\prime}}, \partial_{1^{\prime}}^{1^{\prime}}\right)=\left(\tilde{\partial}, \partial_{0}, \partial_{2}, \partial\right)$. If the field equations are satisfied then the Euclidean slice of $\mathcal{M}$ is equipped with three integrable complex structures given by $J_{i}:=\left\{i\left(\partial_{2}-\partial_{0}\right),(\partial-\tilde{\partial}),\left(\partial_{2}+\partial_{0}\right)\right\}$ and three symplectic structures $\omega_{i}=\left\{(i(\alpha-\tilde{\alpha}), i \omega,(\alpha+\tilde{\alpha})\}\right.$ compatible with the $J_{i}$. It is therefore a hyper-Kähler manifold. 


\subsection{The ASD condition and heavenly equations}

Part of the residual gauge freedom in (2.11) is fixed by selecting one of Plebański's null coordinate systems.

1. Equations (2.13) and (2.14) imply the existence of a coordinate system

$$
(w, z, \tilde{w}, \tilde{z})=:\left(w^{A}, \tilde{w}^{A}\right)
$$

and a complex-valued function $\Omega$ such that

$$
\partial_{A A^{\prime}}=\left(\begin{array}{cc}
\Omega_{w \tilde{w}} \partial_{\tilde{z}}-\Omega_{w \tilde{z}} \partial_{\tilde{w}} & \partial_{w} \\
\Omega_{z \tilde{w}} \partial_{\tilde{z}}-\Omega_{z \tilde{z}} \partial_{\tilde{w}} & \partial_{z}
\end{array}\right)=\left(\begin{array}{ll}
\frac{\partial^{2} \Omega}{\partial w^{A} \partial \tilde{w}^{B}} \frac{\partial}{\partial \tilde{w}_{B}} & \frac{\partial}{\partial w^{A}}
\end{array}\right) .
$$

Equation (2.12) yields the first heavenly equation

$$
\Omega_{w \tilde{z}} \Omega_{z \tilde{w}}-\Omega_{w \tilde{w}} \Omega_{z \tilde{z}}=1 \text { or } \frac{1}{2} \frac{\partial^{2} \Omega}{\partial w_{A} \partial \tilde{w}_{B}} \frac{\partial^{2} \Omega}{\partial w^{A} \partial \tilde{w}^{B}}=1 .
$$

The dual tetrad is

$$
e^{A 1^{\prime}}=\mathrm{d} w^{A}, \quad e^{A 0^{\prime}}=\frac{\partial^{2} \Omega}{\partial w_{A} \partial \tilde{w}_{B}} \mathrm{~d} \tilde{w}_{B}
$$

with the flat solution $\Omega=w^{A} \tilde{w}_{A}$. The only nontrivial part of $\Sigma^{A^{\prime} B^{\prime}}$ is $\Sigma^{0^{\prime} 1^{\prime}}=\partial \tilde{\partial} \Omega$ so that $\Omega$ is a Kähler scalar. The Lax pair for the first heavenly equation is

$$
\begin{aligned}
& L_{0}:=\Omega_{w \tilde{w}} \partial_{\tilde{z}}-\Omega_{w \tilde{z}} \partial_{\tilde{w}}-\lambda \partial_{w}, \\
& L_{1}:=\Omega_{z \tilde{w}} \partial_{\tilde{z}}-\Omega_{z \tilde{z}} \partial_{\tilde{w}}-\lambda \partial_{z} .
\end{aligned}
$$

Equations $L_{0} \Psi=L_{1} \Psi=0$ have solutions provided that $\Omega$ satisfies the first heavenly equation (2.18). Here $\Psi$ is a function on $\mathcal{F}$.

2. Alternatively equations (2.12) and (2.13) imply the existence of a complex-valued function $\Theta$ and coordinate system $(w, z, x, y)=:\left(w^{A}, x_{A}\right), w^{A}$ as above, such that

$$
\partial_{A A^{\prime}}=\left(\begin{array}{cc}
\partial_{y} & \partial_{w}+\Theta_{y y} \partial_{x}-\Theta_{x y} \partial_{y} \\
-\partial_{x} & \partial_{z}-\Theta_{x y} \partial_{x}+\Theta_{x x} \partial_{y}
\end{array}\right)=\left(\begin{array}{cc}
\frac{\partial}{\partial x^{A}} & \frac{\partial}{\partial w^{A}}+\frac{\partial^{2} \Theta}{\partial x^{A} \partial x^{B}} \frac{\partial}{\partial x_{B}}
\end{array}\right) .
$$

As a consequence of (2.14) $\Theta$ satisfies second heavenly equation

$$
\Theta_{x w}+\Theta_{y z}+\Theta_{x x} \Theta_{y y}-\Theta_{x y}^{2}=0 \text { or } \frac{\partial^{2} \Theta}{\partial w^{A} \partial x_{A}}+\frac{1}{2} \frac{\partial^{2} \Theta}{\partial x^{B} \partial x^{A}} \frac{\partial^{2} \Theta}{\partial x_{B} \partial x_{A}}=0 .
$$

The dual frame is given by

$$
e^{A 0^{\prime}}=\mathrm{d} x^{A}+\frac{\partial^{2} \Theta}{\partial x^{B} \partial x_{A}} \mathrm{~d} w^{B}, \quad e^{A 1^{\prime}}=\mathrm{d} w^{A}
$$

with $\Theta=0$ defining the flat metric. The Lax pair corresponding to $(2.22)$ is

$$
\begin{aligned}
& L_{0}=\partial_{y}-\lambda\left(\partial_{w}-\Theta_{x y} \partial_{y}+\Theta_{y y} \partial_{x}\right), \\
& L_{1}=\partial_{x}+\lambda\left(\partial_{z}+\Theta_{x x} \partial_{y}-\Theta_{x y} \partial_{x}\right) .
\end{aligned}
$$

Both heavenly equations were originally derived by Plebański [25 from the formulation (2.16). The closure condition is used, via Darboux's theorem, to introduce $\omega^{A}$, canonical coordinates on the spin bundle, holomorphic around $\lambda=0$ such that the two-form (2.15) is $\Sigma(\lambda)=\mathrm{d}_{h} \omega^{A} \wedge \mathrm{d}_{h} \omega_{A}$. The various forms of the heavenly equations can be obtained by adapting different coordinates and gauges to these forms.

\section{The recursion operator}

In $\S \S 3.1$ the recursion operator $R$ for the anti-self-dual Einstein vacuum equations is constructed. In $\$ \$ 3.2$ then show that the generating function for $R^{i} \phi$ is automatically a twistor function, and is in fact a $C$ ech representative for $\phi$. It is shown that $R$ acts on such a twistor function by multiplication. A similar application to the coordinates used in the heavenly equations yields the coordinate description of the twistor space starting. In $\S \$ 3.3$ we show how that the action of the recursion operator on space-time corresponds to multiplication of the corresponding twistor functions by $\lambda$. In $\S \S 3.4$ the algebra of hidden symmetries of the second heavenly equation is constructed by applying the recursion operator to the explicit symmetries. In $\S \S 3.5, R$ is used to build a higher valence Killing spinors corresponding to hidden symmetries. In the last subsections examples of the use of the recursion operator are given. 


\subsection{The recursion relations}

The recursion operator $R$ is a map from the space of linearised perturbations of the ASDVE equations to itself. This can be used to construct the ASDVE hierarchy whose higher flows are generated by acting on one of the coordinate flows with the recursion operator $R$.

We will identify the space of linearised perturbations to the ASDVE equations with solutions to the background coupled wave equations in two ways as follows.

Lemma 3.1 Let $\square_{\Omega}$ and $\square_{\Theta}$ denote wave operators on the ASD background determined by $\Omega$ and $\Theta$ respectively. Linearised solutions to (2.18) and (2.22) satisfy

$$
\square_{\Omega} \delta \Omega=0, \quad \square_{\Theta} \delta \Theta=0 .
$$

Proof. In both cases $\square_{g}=\nabla_{A 1^{\prime}} \nabla^{A} 0_{0^{\prime}}$ since

$$
\square_{g}=\frac{1}{\sqrt{g}} \partial_{a}\left(g^{a b} \sqrt{g} \partial_{b}\right)=g^{a b} \partial_{a} \partial_{b}+\left(\partial_{a} g^{a b}\right) \partial_{b}
$$

but $\partial_{a} g^{a b}=0$ for both heavenly coordinate systems. For the first equation $(\partial \tilde{\partial}(\Omega+\delta \Omega))^{2}=\nu$ implies

$$
0=(\partial \tilde{\partial} \Omega \wedge \partial \tilde{\partial}) \delta \Omega=\mathrm{d}(\partial \tilde{\partial} \Omega \wedge(\partial-\tilde{\partial}) \delta \Omega)=\mathrm{d} * \mathrm{~d} \delta \Omega .
$$

Here $*$ is the Hodge star operator corresponding to $g$. For the second equation we make use of the tetrad (2.21) and perform coordinate calculations.

From now on we identify tangent spaces to the spaces of solutions to $(2.18)$ and $(2.22)$ with the space of solutions to the curved background wave equation, $\mathcal{W}_{g}$. We will define the recursion operator on the space $\mathcal{W}_{g}$.

The above lemma shows that we can consider a linearised perturbation as an element of $\mathcal{W}_{g}$ in two ways. These two will be related by the square of the recursion operator. The linearised vacuum metrics corresponding to $\delta \Omega$ and $\delta \Theta$ are

$$
h_{A A^{\prime} B B^{\prime}}^{I}=\iota_{\left(A^{\prime} O_{\left.B^{\prime}\right)}\right.} \nabla_{\left(A 1^{\prime}\right.} \nabla_{B) 0^{\prime}} \delta \Omega, \quad h_{A A^{\prime} B B^{\prime}}^{I I}=o_{A^{\prime}} O_{B^{\prime}} \nabla_{A 0^{\prime}} \nabla_{B 0^{\prime}} \delta \Theta .
$$

where $o^{A^{\prime}}=(1,0)$ and $\iota^{A^{\prime}}=(0,1)$ are the constant spin frame associated to the null tetrads given above. Given $\phi \in \mathcal{W}_{g}$ we use the first of these equations to find $h^{I}$. If we put the perturbation obtained in this way on the LHS of the second equation and add an appropriate gauge term we obtain $\phi^{\prime}$ - the new element of $\mathcal{W}_{g}$ that provides the $\delta \Theta$ which gives rise to

$$
h_{a b}^{I I}=h_{a b}^{I}+\nabla_{(a} V_{b)} .
$$

To extract the recursion relations we must find $V$ such that $h^{I} A A^{\prime} B B^{\prime}-\nabla_{\left(A A^{\prime}\right.} V_{\left.B B^{\prime}\right)}=o_{A^{\prime}} O_{B^{\prime}} \chi_{A B}$. Take $V_{B B^{\prime}}=o_{B^{\prime}} \nabla_{B 1^{\prime}} \delta \Omega$, which gives

$$
\nabla_{\left(A A^{\prime}\right.} V_{\left.B B^{\prime}\right)}=-\iota_{\left(A^{\prime} O_{\left.B^{\prime}\right)}\right.} \nabla_{\left(A 0^{\prime}\right.} \nabla_{B) 1^{\prime}} \delta \Omega+o_{A^{\prime} O_{B^{\prime}}} \nabla_{A 1^{\prime}} \nabla_{B 1^{\prime}} \delta \Omega .
$$

This reduces $(3.26)$ to

$$
\nabla_{A 1^{\prime}} \nabla_{B 1^{\prime}} \phi=\nabla_{A 0^{\prime}} \nabla_{B 0^{\prime}} \phi^{\prime}
$$

Definition 3.2 Define the recursion operator $R: \mathcal{W}_{g} \longrightarrow \mathcal{W}_{g}$ by

$$
\iota^{A^{\prime}} \nabla_{A A^{\prime}} \phi=o^{A^{\prime}} \nabla_{A A^{\prime}} R \phi
$$

so formally $R=\left(\nabla_{A 0^{\prime}}\right)^{-1} \circ \nabla_{A 1^{\prime}}$ (no summation over the index $A$ ).

\section{Remarks:}

- From (3.28) and from (2.11) it follows that if $\phi$ belongs to $\mathcal{W}_{g}$ then so does $R \phi$.

- If $R^{2} \delta \Omega=\delta \Theta$ then $\delta \Omega$ and $\delta \Theta$ correspond to the same variation in the metric up to gauge.

- The operator $\phi \mapsto \nabla_{A 0^{\prime}} \phi$ is over-determined, and its consistency follows from the wave equation on $\phi$.

- This definition is formal in that in order to invert the operator $\phi \mapsto \nabla_{A 0^{\prime}} \phi$ we need to specify boundary conditions. 
To summarize:

Proposition 3.3 Let $\mathcal{W}_{g}$ be the space of solutions of the wave equation on the curved ASD background given by $g$.

(i) Elements of $\mathcal{W}_{g}$ can be identified with linearised perturbations of the heavenly equations.

(ii) There exists a (formal) map $R: \mathcal{W}_{g} \longrightarrow \mathcal{W}_{g}$ given by (3.28).

The recursion operator can be generalised to act on solutions to the higher helicity Zero Rest-Mass equations on the ASD vacuum backgrounds [10 by using Herz potentials. We restrict ourselves to the gauge invariant case of left-handed neutrino field $\psi_{A}$ on a heavenly background. First note that any solution of

$$
\nabla^{A A^{\prime}} \psi_{A}=0
$$

must be of the form $\nabla_{A 0^{\prime}} \phi$ where $\phi \in \mathcal{W}_{g}$. Define the recursion relations

$$
\mathcal{R} \psi_{A}:=\nabla_{A 0^{\prime}} R \phi .
$$

It is easy to see that $\mathcal{R}$ maps solutions into solutions, although again the definition is formal in that boundary conditions are required to eliminate the ambiguities. A conjugate recursion operator $\mathcal{R}$ will play a role in the Hamiltonian formulation in Section 5 .

\subsection{The recursion operator and twistor functions}

A twistor function $f$ can be pulled back to the correspondence space $F$. A function $f$ on $F$ descends to twistor space iff $L_{A} f=0$.

Given $\phi \in \mathcal{W}_{g}$, define, for $i \in \mathbb{Z}$, a hierarchy of linear fields, $\phi_{i} \equiv R^{i} \phi_{0}$. Put $\Psi=\sum_{-\infty}^{\infty} \phi_{i} \lambda^{i}$ and observe that the recursion equations are equivalent to $L_{A} \Psi=0$. Thus $\Psi$ is a function on the twistor space $\mathcal{P} \mathcal{T}$. Conversely every solution of $L_{A} \Psi=0$ defined on a neighbourhood of $|\lambda|=1$ can be expanded in a Laurent series in $\lambda$ with the coefficients forming a series of elements of $\mathcal{W}_{g}$ related by the recursion operator. The function $\Psi$, when multiplied by $1 /\left(\pi_{0^{\prime}} \pi_{1^{\prime}}\right)$, is a Cech representative of the element of $H^{1}(\mathcal{P} \mathcal{T}, \mathcal{O}(-2))$ that corresponds to the solution of the wave equation $\phi$ under the Penrose transform (i.e. by integration around $|\lambda|=1$ ). The ambiguity in the inversion of $\nabla_{A 0^{\prime}}$ means that there are many such functions $\Psi$ that can be obtained from a given $\phi$. However, they are all equivalent as cohomology classes.

It is clear that a series corresponding to $R \phi$ is the function $\lambda^{-1} \Psi$. As noted before, $R$ is not completely well defined when acting on $\mathcal{W}_{g}$ because of the ambiguity in the inversion of $\nabla_{A 0^{\prime}}$. However, the definition $R \Psi=\Psi / \lambda$ is well defined as a twistor function on $\mathcal{P} \mathcal{T}$, but the problem resurfaces when one attempts to treat $\Psi(\lambda)$ as a representative of a cohomology class since pure gauge elements of the first sheaf cohomology group $H^{1}(\mathcal{P} \mathcal{T}, \mathcal{O}(-2))$ are mapped to functions defining a non-trivial element of the cohomology. Note, however, that with the definition $R \Psi=\Psi / \lambda$, the action of $R$ is well defined on twistor functions and can be iterated without ambiguity.

We can in this way build coordinate charts on twistor space from those on space-time arising from the choices in the Plebanski reductions. Put $\omega_{0}^{A}=w^{A}=(w, z)$; the surfaces of constant $\omega_{0}^{A}$ are twistor surfaces. We have that $\nabla^{A}{ }_{0^{\prime}} \omega_{0}^{B}=0$ so that in particular $\nabla_{A 1^{\prime}} \nabla^{A}{ }_{0^{\prime}} \omega_{0}^{B}=0$ and if we define $\omega_{i}^{A}=R^{i} \omega_{0}^{A}$ then we can choose $\omega_{i}^{A}=0$ for negative $i$. We define

$$
\omega^{A}=\sum_{i=0}^{\infty} \omega_{i}^{A} \lambda^{i}
$$

We can similarly define $\tilde{\omega}^{A}$ by $\tilde{\omega}_{0}^{A}=\tilde{w}^{A}$ and choose $\tilde{\omega}_{i}^{A}=0$ for $i>0$. Note that $\omega^{A}$ and $\tilde{\omega}^{A}$ are solutions of $L_{A}$ holomorphic around $\lambda=0$ and $\lambda=\infty$ respectively and they can be chosen so that they extend to a neighbourhood of the unit disc and a neighbourhood of the complement of the unit disc and can therefore be used to provide a patching description of the twistor space.

\subsection{The Penrose transform of linearised deformations and the recursion op- erator}

The recursion operator acts on linearised perturbations of the ASDVE equations. Under the twistor correspondence, these correspond to linearised holomorphic deformations of (part of) $\mathcal{P} \mathcal{T}$.

Cover $\mathcal{P} \mathcal{T}$ by two sets, $U$ and $\tilde{U}$ with $|\lambda|<1+\epsilon$ on $U$ and $|\lambda|>1-\epsilon$ on $\tilde{U}$ with $\left(\omega^{A}, \lambda\right)$ coordinates on $U$ and $\left(\tilde{\omega}^{A}, \lambda^{-1}\right)$ on $\tilde{U}$. The twistor space $\mathcal{P} \mathcal{T}$ is then determined by the transition function $\tilde{\omega}^{B}=$ $\tilde{\omega}^{B}\left(\omega^{A}, \pi_{A^{\prime}}\right)$ on $U \cap \tilde{U}$ which preserves the fibrewise 2 -form, $\left.\mathrm{d} \omega^{A} \wedge \mathrm{d} \omega_{A}\right|_{\lambda=\text { const. }}=\left.\mathrm{d} \tilde{\omega}^{A} \wedge \mathrm{d} \tilde{\omega}_{A}\right|_{\lambda=\text { const. }}$. 
Infinitesimal deformations are given by elements of $H^{1}(\mathcal{P} \mathcal{T}, \boldsymbol{\Theta})$, where $\boldsymbol{\Theta}$ denotes a sheaf of germs of holomorphic vector fields. Let

$$
Y=f^{A}\left(\omega^{B}, \pi_{B^{\prime}}\right) \frac{\partial}{\partial \omega^{A}}
$$

defined on the overlap $U \cap \tilde{U}$ and define a class in $H^{1}(\mathcal{P} \mathcal{T}, \boldsymbol{\Theta})$ that preserves the fibration $\mathcal{P} \mathcal{T} \mapsto \mathbb{C} \mathbb{P}^{1}$. The corresponding infinitesimal deformation is given by

$$
\tilde{\omega}^{A}\left(\omega^{A}, \pi_{A^{\prime}}, t\right)=(1+t Y)\left(\tilde{\omega}^{A}\right)+O\left(t^{2}\right) .
$$

From the globality of $\Sigma(\lambda)=\mathrm{d} \omega^{A} \wedge \mathrm{d} \omega_{A}$ it follows that $Y$ is a Hamiltonian vector field with a Hamiltonian $f \in H^{1}(\mathcal{P} \mathcal{T}, \mathcal{O}(2))$ with respect to the symplectic structure $\Sigma$. A finite deformation is given by integrating

$$
\frac{\mathrm{d} \tilde{\omega}^{B}}{\mathrm{~d} t}=\varepsilon^{B A} \frac{\partial f}{\partial \tilde{\omega}^{A}} .
$$

from $t=0$ to 1 . Infinitesimally we can put

$$
\delta \tilde{\omega}^{A}=\frac{\partial \delta f}{\partial \tilde{\omega}_{A}} .
$$

If the ASD metric is determined by $\Theta$ and then $\varepsilon^{B A} \partial \delta f / \partial \omega^{B}$, (or more simply $\delta f$ ) is a linearised deformation corresponding to $\delta \Theta \in \mathcal{W}_{g}$.

The recursion operator acts on linearised deformations as follows

Proposition 3.4 Let $R$ be the recursion operator defined by (3.28). Its twistor counterpart is the multiplication operator

$$
R \delta f=\frac{\pi_{1^{\prime}}}{\pi_{0^{\prime}}} \delta f=\lambda^{-1} \delta f
$$

[Note that $R$ acts on $\delta f$ without ambiguity; the ambiguity in boundary condition for the definition of $R$ on space-time is absorbed into the choice of explicit representative for the cohomology class determined by $\delta f$.

Proof. Pull back $\delta f$ to the primed spin bundle on which it is a coboundary so that

$$
\delta f\left(\pi_{A^{\prime}}, x^{a}\right)=h\left(\pi_{A^{\prime}}, x^{a}\right)-\tilde{h}\left(\pi_{A^{\prime}}, x^{a}\right)
$$

where $h$ and $\tilde{h}$ are holomorphic on $U$ and $\tilde{U}$ respectively (here we abuse notation and denote by $U$ and $\tilde{U}$ the open sets on the spin bundle that are the preimage of $U$ and $\tilde{U}$ on twistor space). A choice for the splitting (3.34) is given by

$$
\begin{aligned}
h & =\frac{1}{2 \pi i} \oint_{\Gamma} \frac{\left(\pi^{A^{\prime}} o_{A^{\prime}}\right)^{3}}{\left(\rho^{C^{\prime}} \pi_{C^{\prime}}\right)\left(\rho^{B^{\prime}} o_{B^{\prime}}\right)^{3}} \delta f\left(\rho_{E^{\prime}}\right) \rho_{D^{\prime}} \mathrm{d} \rho^{D^{\prime}}, \\
\tilde{h} & =\frac{1}{2 \pi i} \oint_{\tilde{\Gamma}} \frac{\left(\pi^{A^{\prime}} o_{A^{\prime}}\right)^{3}}{\left(\rho^{C^{\prime}} \pi_{C^{\prime}}\right)\left(\rho^{B^{\prime} o_{B^{\prime}}}\right)^{3}} \delta f\left(\rho_{E^{\prime}}\right) \rho_{D^{\prime}} \mathrm{d} \rho^{D^{\prime}} .
\end{aligned}
$$

Here $\rho_{A^{\prime}}$ are homogeneous coordinates of $\mathbb{C P}^{1}$ pulled back to the spin bundle. The contours $\Gamma$ and $\tilde{\Gamma}$ are homologous to the equator of $\mathbb{C P}^{1}$ in $U \cap \tilde{U}$ and are such that $\Gamma-\tilde{\Gamma}$ surrounds the point $\rho_{A^{\prime}}=\pi_{A^{\prime}}$.

The functions $h$ and $\tilde{h}$ are homogeneous of degree 1 in $\pi_{A^{\prime}}$ and do not descend to $\mathcal{P} \mathcal{T}$, whereas their difference does so that

$$
\pi^{A^{\prime}} \nabla_{A A^{\prime}} h=\pi^{A^{\prime}} \nabla_{A A^{\prime}} \tilde{h}=\pi^{A^{\prime}} \pi^{B^{\prime}} \pi^{C^{\prime}} \Sigma_{A A^{\prime} B^{\prime} C^{\prime}}
$$

where the first equality shows that the LHS is global with homogeneity degree 2 and implies the second equality for some $\Sigma_{A A^{\prime} B^{\prime} C^{\prime}}$ which will be the third potential for a linearised ASD Weyl spinor. $\Sigma_{A A^{\prime} B^{\prime} C^{\prime}}$ is in general defined modulo terms of the form $\nabla_{A\left(A^{\prime}\right.} \gamma_{\left.B^{\prime} C^{\prime}\right)}$ but this gauge freedom is partially fixed by choosing the integral representation above; $h$ vanishes to third order at $\pi_{A^{\prime}}=o_{A^{\prime}}$ and direct differentiation, using $\nabla_{A A^{\prime}} \delta f=\rho_{A^{\prime}} \delta f_{A}$ for some $\delta f_{A}$, gives $\Sigma_{A A^{\prime} B^{\prime} C^{\prime}}=o_{A^{\prime}} O_{B^{\prime}} O_{C^{\prime}} \nabla_{A 0^{\prime}} \delta \Theta$ where

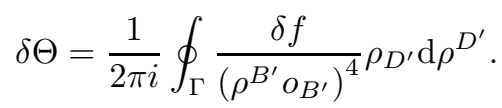

This is consistent with the Plebanski gauge choices (there is also a gauge freedom in $\delta \Theta$ arising from cohomology freedom in $\delta f$ which we shall describe in the next subsection.) The condition $\nabla_{A\left(D^{\prime}\right.} \Sigma^{A} A_{\left.A^{\prime} B^{\prime} C^{\prime}\right)}=0$ follows from equation (3.36) which, with the Plebański gauge choice, implies $\delta \Theta \in \mathcal{W}_{g}$. Thus we obtain a twistor integral formula for the linearisation of the second heavenly equation. 
Now recall formula (3.28) defining $R$. Let $R \delta f$ be the twistor function corresponding to $R \delta \Theta$ by (3.37). The recursion relations yield

$$
\oint_{\Gamma} \frac{R \delta f_{A}}{\left(\rho^{B^{\prime}} o_{B^{\prime}}\right)^{3}} \rho_{D^{\prime}} \mathrm{d} \rho^{D^{\prime}}=\oint_{\Gamma} \frac{\delta f_{A}}{\left(\rho^{B^{\prime}} o_{B^{\prime}}\right)^{2}\left(\rho^{B^{\prime}} \iota_{B^{\prime}}\right)} \rho_{D^{\prime}} \mathrm{d} \rho^{D^{\prime}}
$$

so $R \delta f=\lambda^{-1} \delta f$.

Let $\delta \Omega$ be the linearisation of the first heavenly potential. From $R^{2} \delta \Omega=\delta \Theta$ it follows that

$$
\delta \Omega=\frac{1}{2 \pi i} \oint_{\Gamma} \frac{\delta f}{\left(\rho_{A^{\prime} O^{A^{\prime}}}\right)^{2}\left(\rho_{B^{\prime}} \iota^{B^{\prime}}\right)^{2}} \rho_{C^{\prime}} \mathrm{d} \rho^{C^{\prime}} .
$$

\subsection{Hidden symmetry algebra}

The ASDVE equations in the Plebański forms have a residual coordinate symmetry. This consists of area preserving diffeomorphisms in the $w^{A}$ coordinates together with some extra transformations that depend on whether one is reducing to the first or second form. By regarding the infinitesimal forms of these transformations as linearised perturbations and acting on them using the recursion operator, the coordinate (passive) symmetries can be extended to give 'hidden' (active) symmetries of the heavenly equations. Formulae (3.37) and (3.33) can be used to recover the known relations (see for example [28]) of the hidden symmetry algebra of the heavenly equations. We deal with the second equation as the case of the first equation was investigated by other methods [21].

Let $M$ be a volume preserving vector field on $\mathcal{M}$. Define $\delta_{M}^{0} \nabla_{A A^{\prime}}:=\left[M, \nabla_{A A^{\prime}}\right]$. This is a pure gauge transformation corresponding to addition of $\mathcal{L}_{M} g$ to the space-time metric and preserves the field equations. Note that

$$
\left[\delta_{M}^{0}, \delta_{N}^{0}\right] \nabla_{A A^{\prime}}:=\delta_{[M, N]}^{0} \nabla_{A A^{\prime}} .
$$

Once a Plebański coordinate system and reduced equations have been obtained, the reduced equation will not be invariant under all the $\operatorname{SDiff}(\mathcal{M})$ transformations. The second form will be preserved if we restrict ourselves to transformations which preserve the SD two-forms $\Sigma^{1^{\prime} 1^{\prime}}=\mathrm{d} w_{A} \wedge \mathrm{d} w^{A}$ and $\Sigma^{0^{\prime} 1^{\prime}}=\mathrm{d} x_{A} \wedge \mathrm{d} w^{A}$. The conditions $\mathcal{L}_{M} \Sigma^{0^{\prime} 0^{\prime}}=\mathcal{L}_{M} \Sigma^{0^{\prime} 1^{\prime}}=0$ imply that $M$ is given by

$$
M=\frac{\partial h}{\partial w_{A}} \frac{\partial}{\partial w^{A}}+\left(\frac{\partial g}{\partial w_{A}}-x^{B} \frac{\partial^{2} h}{\partial w_{A} \partial w^{B}}\right) \frac{\partial}{\partial x^{A}}
$$

where $h=h\left(w^{A}\right)$ and $g=g\left(w^{A}\right)$. The space-time is now viewed as a cotangent bundle $\mathcal{M}=T^{*} \mathcal{N}^{2}$ with $w^{A}$ being coordinates on a two-dimensional complex manifold $\mathcal{N}^{2}$. The full $\operatorname{SDiff}(\mathcal{M})$ symmetry breaks down to the semi-direct product of $\operatorname{SDiff}\left(\mathcal{N}^{2}\right)$, which acts on $\mathcal{M}$ by a Lie lift, with $\Gamma\left(\mathcal{N}^{2}, \mathcal{O}\right)$ which acts on $\mathcal{M}$ by translations of the zero section by the exterior derivatives of functions on $\mathcal{N}^{2}$. Let $\delta_{M} \Theta$ correspond to $\delta_{M}^{0} \nabla_{A A^{\prime}}$ by

$$
\delta_{M}^{0} \nabla_{A 1^{\prime}}=\frac{\partial^{2} \delta_{M} \Theta}{\partial x^{A} \partial x^{B}} \frac{\partial}{\partial x_{B}} .
$$

The 'pure gauge' elements are

$$
\begin{aligned}
\delta_{M}^{0} \Theta= & F+x_{A} G^{A}+x_{A} x_{B} \frac{\partial^{2} g}{\partial w_{A} \partial w_{B}}+x_{A} x_{B} x_{C} \frac{\partial^{3} h}{\partial w_{A} \partial w_{B} \partial w_{C}} \\
& +\frac{\partial g}{\partial w_{A}} \frac{\partial \Theta}{\partial x^{A}}+\frac{\partial h}{\partial w_{A}} \frac{\partial \Theta}{\partial w^{A}}-x^{B} \frac{\partial^{2} h}{\partial w_{A} \partial w^{B}} \frac{\partial \Theta}{\partial x^{A}}
\end{aligned}
$$

where $F, G^{A}, g, h$ are functions of $w^{B}$ only.

The above symmetries can be seen to arise from symmetries on twistor space as follows. Since we have the symplectic form $\Sigma=\mathrm{d} \omega^{A} \wedge \mathrm{d} \omega_{A}$ on the fibres of $\mu: \mathcal{P} \mathcal{T} \longrightarrow \mathbb{C P}^{1}$, a symmetry is a holomorphic diffeomorphism of the set $U$ that restricts to a canonical transformation on each fibre. Let $H=H\left(x^{a}, \lambda\right)=$

$\sum_{i=0}^{\infty} h_{i} \lambda^{i}$ be the Hamiltonian for an infinitesimal such transformation pulled back to the projective spin bundle. The functions $h_{i}$ depend on space time coordinates only. In particular $h_{0}$ and $h_{1}$ give $h$ and $g$ from the previous construction (3.38). This can be seen by calculating how $\Theta$ transforms if $\omega^{A}=w^{A}+\lambda x^{A}+\lambda^{2} \partial \Theta / \partial x_{A}+\ldots \longrightarrow \hat{\omega}^{A}$. Now $\Theta$ is treated as an object on the first jet bundle of a fixed fibre of $\mathcal{P} \mathcal{T}$ and it determines the structure of the second jet.

These symmetries take a solution to an equivalent solution. The recursion operator can be used to define an algebra of 'hidden symmetries' that take one solution to a different one as follows. 
Let $\delta_{M}^{0} \Theta$ be an expression of the form (3.38) which also satisfies $\square_{g} \delta_{M}^{0} \Theta=0$. We set

$$
\delta_{M}^{i} \Theta:=R^{i} \delta_{M} \Theta \in \mathcal{W}_{g} .
$$

Proposition 3.5 Generators of the hidden symmetry algebra of the second heavenly equation satisfy the relation

$$
\left[\delta_{M}^{i}, \delta_{N}^{j}\right]=\delta_{[M, N]}^{i+j} .
$$

Proof. This can be proved directly by showing that the ambiguities in $R$ can be chosen so that $R \circ \delta_{M}=$ $\delta_{M} \circ R$. It is perhaps more informative to prove it by its action on twistor functions.

Let $\delta_{M}^{i} f$ be the twistor function corresponding to $\delta_{M}^{i} \Theta$ (by (3.37)) treated as an element of $\Gamma(U \cap$ $\tilde{U}, \mathcal{O}(2))$ rather than $H^{1}(\mathcal{P} \mathcal{T}, \mathcal{O}(2))$. Define $\left[\delta_{M}^{i}, \delta_{N}^{j}\right]$ by

$$
\left[\delta_{M}^{i}, \delta_{N}^{j}\right] \Theta:=\frac{1}{2 \pi i} \oint \frac{\left\{\delta_{M}^{i} f, \delta_{N}^{j} f\right\}}{\left(\pi_{0^{\prime}}\right)^{4}} \pi_{A^{\prime}} \mathrm{d} \pi^{A^{\prime}}
$$

where the Poisson bracket is calculated with respect to a canonical Poisson structure on $\mathcal{P} \mathcal{T}$. From Proposition (3.33) it follows that

$$
\left[\delta_{M}^{i}, \delta_{N}^{j}\right] \Theta=\frac{1}{2 \pi i} \oint \lambda^{-i-j} \frac{\left\{\delta_{M} f, \delta_{N} f\right\}}{\left(\pi_{0^{\prime}}\right)^{4}} \pi_{A^{\prime}} \mathrm{d} \pi^{A^{\prime}}=R^{i+j} \delta_{[M, N]} \Theta
$$

as required.

\subsection{Recursion procedure for Killing spinors}

Let $(\mathcal{M}, g)$ be an ASD vacuum space. We say that $L_{A_{1}^{\prime} \ldots A_{n}^{\prime}}$ is a Killing spinor of type $(0, n)$ if

$$
\nabla_{\left(A^{\prime}\right.}^{A} L_{\left.B_{1}^{\prime} \ldots B_{n}^{\prime}\right)}=0
$$

Killing spinors of type $(0, n)$ give rise to Killing spinors of type $(1, n-1)$ by

$$
\nabla_{A^{\prime}}^{A} L_{B_{1}^{\prime} \ldots B_{n}^{\prime}}=\varepsilon_{A^{\prime}\left(B_{1}^{\prime}\right.} K_{\left.B_{2}^{\prime} \ldots B_{n}^{\prime}\right)} .
$$

In an $\mathrm{ASD}$ vacuum, $K^{B B_{2}^{\prime} \ldots B_{n}^{\prime}}$ is also a Killing spinor

$$
\nabla_{\left(A^{\prime}\right.}^{(A} K^{B)}{ }_{\left.B_{1}^{\prime} \ldots B_{n}^{\prime}\right)}=0 .
$$

Put (for $i=0, \ldots, n)$

$$
L_{i}:=\iota^{B_{1}^{\prime}} \ldots \iota^{B_{i}^{\prime}} O^{B_{i+1}^{\prime}} \ldots o^{B_{n}^{\prime}} L_{B_{1}^{\prime} \ldots B_{n}^{\prime}},
$$

and contract (3.40) with $\iota^{B_{1}^{\prime}} \ldots \iota^{B_{i}^{\prime}} O^{B_{i+1}^{\prime}} \ldots o^{B_{n+1}^{\prime}}$ to obtain

$$
i \nabla_{A 1^{\prime}} L_{i-1}=-(n-i+1) \nabla_{A 0^{\prime}} L_{i}, \quad i=0, \ldots, n-1 .
$$

We make use of the recursion relations (3.28):

$$
\frac{-i}{n+1-i} R\left(L_{i-1}\right)=L_{i} .
$$

This leads to a general formula for Killing spinors (with $\nabla_{A 0^{\prime}} L_{0}=0$ )

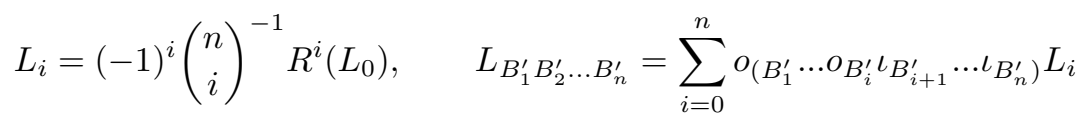

and equation (3.40) is then satisfied iff $R^{-1} L_{0}=R L_{n}=0$. 


\subsection{Example 1}

Let us demonstrate how to use the recursion procedure to find metrics with hidden symmetries. Let $\partial_{t_{n}} \Omega:=\phi_{n}$ be a linearisation of the first heavenly equation. We have $R: z \longrightarrow \Omega_{w}=\partial_{t_{1}} \Omega$. Look for solutions to (2.18) with an additional constraint $\partial_{t_{2}} \Omega=0$. The recursion relations (3.28) imply $\Omega_{w z}=\Omega_{w w}=0$, therefore

$$
\Omega(w, z, \tilde{w}, \tilde{z})=w q(\tilde{w}, \tilde{z})+P(z, \tilde{w}, \tilde{z}) .
$$

The heavenly equation yields $\mathrm{d} q \wedge \mathrm{d} P \wedge \mathrm{d} z=\mathrm{d} \tilde{z} \wedge \mathrm{d} \tilde{w} \wedge \mathrm{d} z$. With the definition $\partial_{z} P=p$ the metric is

$$
\mathrm{d} s^{2}=2 \mathrm{~d} w \mathrm{~d} q+2 \mathrm{~d} z \mathrm{~d} p+f \mathrm{~d} z^{2},
$$

where $f=-2 P_{z z}$. We adopt $(w, z, q, p)$ as a new coordinate system. Heavenly equations imply that $f=f(q, z)$ is an arbitrary function of two variables. These are the null ASD plane wave solutions.

\subsection{Example 2}

Now we shall illustrate the Propositions 3.3 and 3.4 with the example of the Sparling-Tod solution [27. The coordinate formulae for the pull back of twistor functions are:

$$
\begin{aligned}
& \mu^{0}=w+\lambda y-\lambda^{2} \Theta_{x}+\lambda^{3} \Theta_{z}+\ldots, \\
& \mu^{1}=z-\lambda x-\lambda^{2} \Theta_{y}-\lambda^{3} \Theta_{w}+\ldots .
\end{aligned}
$$

Consider

$$
\Theta=\frac{\sigma}{w x+z y}
$$

where $\sigma=$ const. It satisfies both the linear and the nonlinear part of $(2.22)$.

The flat case: First we shall treat (3.43), with $\sigma=1$, as a solution $\phi_{0}$ to the wave equation on the flat background. The recursion relations are

$$
\left(R \phi_{0}\right)_{x}=\frac{y}{(w x+z y)^{2}}, \quad\left(R \phi_{0}\right)_{y}=\frac{-x}{(w x+z y)^{2}} .
$$

They have a solution $\phi_{1}:=R \phi_{0}=(-y / w) \phi_{0}$. More generally we find that

$$
\phi_{n}:=R^{n} \phi_{0}=\left(-\frac{y}{w}\right)^{n} \frac{1}{w x+z y} .
$$

The last formula can be also found using twistor methods. The twistor function corresponding to $\phi_{0}$ is $1 /\left(\mu^{0} \mu^{1}\right)$, where $\mu_{0}=w+\lambda y$ and $\mu_{1}=z-\lambda x$. By Proposition 3.33 the twistor function corresponding to $\phi_{n}$ is $\lambda^{-n} /\left(\mu^{0} \mu^{1}\right)$. This can be seen by applying the formula (3.37) and computing the residue at the pole $\lambda=-w / y$. It is interesting to ask whether any $\phi_{n}$ (apart from $\phi_{0}$ ) is a solution to the heavenly equation. Inserting $\Theta=\phi_{n}$ to (2.22) yields $n=0$ or $n=2$. We parenthetically mention that $\phi_{2}$ yields (by formula (2.23) a metric of type $D$ which is conformal to the Eguchi-Hanson solution.

The curved case. Now let $\Theta$ given by (3.43) determine the curved metric

$$
\mathrm{d} s^{2}=2 \mathrm{~d} w \mathrm{~d} x+2 \mathrm{~d} z \mathrm{~d} y+4 \sigma(w x+z y)^{-3}(w \mathrm{~d} z-z \mathrm{~d} w)^{2} .
$$

The recursion relations

$$
\partial_{y}(R \phi)=\left(\partial_{w}-\Theta_{x y} \partial_{y}+\Theta_{y y} \partial_{x}\right) \phi, \quad-\partial_{x}(R \phi)=\left(\partial_{z}+\Theta_{x x} \partial_{y}-\Theta_{x y} \partial_{x}\right) \phi
$$

are

$$
\begin{aligned}
-\partial_{x}(R \psi) & =\left(\partial_{z}+2 \sigma w(w x+z y)^{-3}\left(w \partial_{x}-z \partial_{y}\right)\right) \psi \\
\partial_{y}(R \psi) & =\left(\partial_{w}+2 \sigma z(w x+z y)^{-3}\left(w \partial_{x}-z \partial_{y}\right)\right) \psi
\end{aligned}
$$

where $\psi$ satisfies

$$
\square_{\Theta} \psi=2\left(\partial_{x} \partial_{w}+\partial_{y} \partial_{z}+2 \sigma(w x+z y)^{-3}\left(z^{2} \partial_{x}{ }^{2}+w^{2} \partial_{y}{ }^{2}-2 w z \partial_{x} \partial_{y}\right)\right) \psi=0 .
$$

One solution to the last equation is $\psi_{1}=(w x+z y)^{-1}$. We apply the recursion relations to find the sequence of linearised solutions

$$
\begin{aligned}
\psi_{2} & =\left(-\frac{y}{w}\right) \frac{1}{w x+z y}, \psi_{3}=-\frac{2}{3} \frac{\sigma}{(w x+z y)^{3}}+\left(-\frac{y}{w}\right)^{2} \frac{1}{w x+z y}, \ldots \\
\psi_{n} & =\sum_{k=0}^{n} A_{(n)}^{k}\left(-\frac{y}{w}\right)^{k}(w x+z y)^{k-n}
\end{aligned}
$$


To find $A_{(n)}^{k}$ note that the recursion relations imply

$$
\begin{aligned}
& R \quad\left(\left(-\frac{y}{w}\right)^{k}(w x+z y)^{j}\right)= \\
& \left.=\left(\left(-\frac{y}{w}\right)-\sigma\left(-\frac{y}{w}\right)^{-1}(w x+z y)^{-2} \frac{k}{j+2}\right)\left(-\frac{y}{w}\right)^{k}(w x+z y)^{j}\right) .
\end{aligned}
$$

This yields a recursive formula

$$
A_{(n+1)}^{k}=A_{(n)}^{k-1}-2 \sigma \frac{k+1}{n-k+1} A_{(n)}^{k+1}, \quad A_{(1)}^{0}=1, \quad A_{(1)}^{1}=0, \quad A_{(n)}^{-1}=0, \quad k=0 \ldots n,
$$

which determines the algebraic (as opposed to the differential) recursion relations between $\psi_{n}$ and $\psi_{n+1}$. It can be checked that functions $\psi_{n}$ indeed satisfy (3.46). Notice that if $\sigma=0$ (flat background) then we recover (3.44). We can also find the inhomogeneous twistor coordinates pulled back to $\mathcal{F}$

$$
\begin{aligned}
\mu^{0} & =w+\lambda y+\sum_{n=0}^{\infty} \sigma \lambda^{n+2} \sum_{k=0}^{n} B_{(n)}^{k} w\left(-\frac{y}{w}\right)^{k}(w x+z y)^{k-n-1}, \\
\mu^{1} & =z-\lambda x+\sum_{n=0}^{\infty} \sigma \lambda^{n+2} \sum_{k=0}^{n} B_{(n)}^{k} z\left(\frac{x}{z}\right)^{k}(w x+z y)^{k-n-1} .
\end{aligned}
$$

where

$$
B_{(n+1)}^{k}=B_{(n)}^{k-1}-2 \sigma \frac{k+1}{n-k+2} B_{(n)}^{k+1}, \quad B_{(1)}^{0}=1, \quad B_{(1)}^{1}=0, \quad B_{(n)}^{-1}=0, \quad k=0 \ldots n .
$$

The polynomials $\mu^{A}$ solve $L_{A}\left(\mu^{B}\right)=0$, where now

$$
\begin{aligned}
& L_{0}=-\lambda \partial_{w}-2 \lambda \sigma z^{2}(w z+z y)^{-3} \partial_{x}+\left(1+2 \lambda \sigma w z(w z+z y)^{-3}\right) \partial_{y} \\
& \left.L_{1}=\lambda \partial_{z}+\left(1-2 \lambda \sigma w z(w z+z y)^{-3}\right) \partial_{x}+2 \lambda \sigma w^{2}(w z+z y)^{-3}\right) \partial_{y} .
\end{aligned}
$$

\section{Hierarchies for the ASD vacuum equations}

The hidden symmetries corresponding to higher flows associated to translations along the coordinate vector fields give 'higher flows' of a hierarchy. This yields a hierarchy of flows of the anti-self-dual Einstein vacuum equations. We first give this for the equations in their second heavenly form but then give the equations in the form of consistency conditions for a Lax system of vector fields generalizing equations 2.11. The nonlinear graviton construction generalizes to give a construction for the corresponding system of equations and is presented in $\S \S 4.2$. In $\S \S 4.3$ the geometric structure of solutions to the truncated hierarchy are explored in further detail. Finally in $\$ \S 4.4$ infinitesimal deformations are studied.

\subsection{Hierarchies for the heavenly equations}

The generators of higher flows are first obtained by applying powers of the recursion operator to the linearised perturbations corresponding to the evolution along coordinate vector fields. This embeds the second heavenly equation into an infinite system of over-determined, but consistent, PDEs (which we will truncate at some arbitrary but finite level). These equations in turn can be naturally embedded into a system of equations that are the consistency conditions for an associated linear system that extends (2.11). We shall discuss here the hierarchy for the second Plebański form; that for the first arises from a different coordinate and gauge choice.

Introduce the coordinates $x^{A i}$, where for $i=0,1, x^{A i}=x^{A A^{\prime}}$ are the original coordinates on $\mathcal{M}$, and for $1<i \leq n, x^{A i}$ are the parameters for the new flows (with $2 n-2$ dimensional parameter space $\mathbb{X}$ ). The propagation of $\Theta$ along these parameters is determined by the recursion relations

$$
\begin{aligned}
\partial_{y}\left(\partial_{B i+1} \Theta\right) & =\left(\partial_{w}-\Theta_{x y} \partial_{y}+\Theta_{y y} \partial_{x}\right) \partial_{B i} \Theta, \\
-\partial_{x}\left(\partial_{B i+1} \Theta\right) & =\left(\partial_{z}+\Theta_{x x} \partial_{y}-\Theta_{x y} \partial_{x}\right) \partial_{B i} \Theta, \\
\text { or } \partial_{A 0}\left(\partial_{B i+1} \Theta\right) & =\left(\partial_{A 1}+\partial_{C 0} \partial_{A 0} \Theta \partial^{C}{ }_{0}\right) \partial_{B i} \Theta .
\end{aligned}
$$

However, we will take the hierarchy to be the system (containing the above when $j=1$ )

$$
\partial_{A i} \partial_{B j-1} \Theta-\partial_{B j} \partial_{A i-1} \Theta+\left\{\partial_{A i-1} \Theta, \partial_{B j-1} \Theta\right\}_{y x}=0, \quad i, j=1 \ldots n \text {. }
$$

Here $\{\ldots, \ldots\}_{y x}$ is the Poisson bracket with respect to the Poisson structure $\partial / \partial x^{A} \wedge \partial / \partial x_{A}=2 \partial_{x} \wedge \partial_{y}$. 
Lemma 4.1 The linear system for equations (4.49) is

$$
L_{A i} s=\left(-\lambda D_{A i+1}+\delta_{A i}\right) s=0, \quad i=0, \ldots, n-1,
$$

where

1. $s:=s\left(x^{A i}, \lambda\right)$ is a function on a spin bundle $\left(a \mathbb{C P}^{1}\right.$-bundle) over $\mathcal{N}=\mathcal{M} \times \mathbb{X}$,

2. $D_{A i+1}:=\partial_{A i+1}+\left[\partial_{A i}, V\right],\left(V=\varepsilon^{A B} \partial_{A 0} \Theta \partial_{B 0}\right)$ and $\delta_{A i}:=\partial_{A i}$ are $4 n$ vector fields on $\mathcal{N}$.

Proof. This follows by direct calculation. The compatibility conditions for (4.50) are:

$$
\begin{gathered}
{\left[D_{A i+1}, D_{B j+1}\right]=0,} \\
{\left[\delta_{A i}, \delta_{B j}\right]=0,} \\
{\left[D_{A i+1}, \delta_{B j}\right]-\left[D_{B j+1}, \delta_{A i}\right]=0 .}
\end{gathered}
$$

It is straightforward to see that equations (4.52) and (4.53) hold identically with the above definitions and (4.51) is equivalent to (4.49).

As a converse to this lemma, we will see in $\S \S 4.2$ using the twistor correspondence, that given the Lax system above, in which the vector fields $D_{A i}$ and $\delta_{A j}$ are volume preserving vector fields, then coordinate and gauge choices can be made so that the Lax system takes on the above form.

\subsubsection{Spinor notation}

The above can also be represented in a spinorial formulation that will be useful later. We introduce the spinor indexed coordinates $x^{A A_{1}^{\prime} \ldots A_{n}^{\prime}}=x^{A\left(A_{1}^{\prime} \ldots A_{n}^{\prime}\right)}$ on $\mathcal{N}$ which correspond to the $x^{A i}$ by

$$
x^{A i}=\left(\begin{array}{c}
n \\
i
\end{array}\right) x^{A A_{1}^{\prime} A_{2}^{\prime} \ldots A_{n}^{\prime}} o_{A_{1}^{\prime}} \ldots o_{A_{i}^{\prime}} \iota_{A_{i+1}^{\prime}} \ldots \iota_{A_{n}^{\prime}}(-1)^{n-i} .
$$

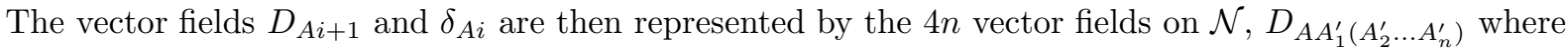

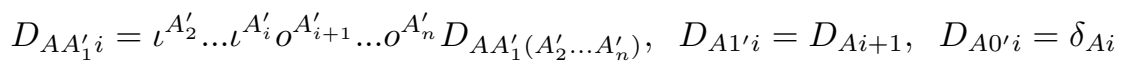

and $L_{A\left(A_{2}^{\prime} \ldots A_{n}^{\prime}\right)}=\pi^{A_{1}^{\prime}} D_{A A_{1}^{\prime}\left(A_{2}^{\prime} \ldots A_{n}^{\prime}\right)}, L_{A i}=\pi^{A_{1}^{\prime}} D_{A A_{1}^{\prime} i}$. In the adopted gauge

$$
D_{A 0^{\prime} A_{2}^{\prime} \ldots A_{n}^{\prime}}=\partial_{A 0^{\prime} A_{2}^{\prime} \ldots A_{n}^{\prime}}, \quad D_{A 1^{\prime} A_{2}^{\prime} \ldots A_{n}^{\prime}}=\partial_{A 1^{\prime} A_{2}^{\prime} \ldots A_{n}^{\prime}}+\left[\partial_{A 0^{\prime} A_{2}^{\prime} \ldots A_{n}^{\prime}}, V\right] .
$$

In what follows we will often be interested in $\nabla_{A\left(A_{1}^{\prime} A_{2}^{\prime} \ldots A_{n}^{\prime}\right)}$, the symmetric part of $D_{A A_{1}^{\prime} A_{2}^{\prime} \ldots A_{n}^{\prime}}$.

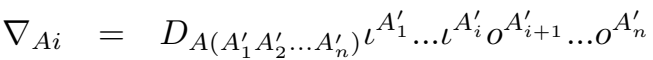

$$
\begin{aligned}
& =\frac{1}{n}\left(i D_{A 1^{\prime} i-1}+(n-i) D_{A 0^{\prime} i}\right)=\partial_{A i}+\frac{i}{n}\left[\partial_{A i-1}, V\right] .
\end{aligned}
$$

Put $D_{A 0^{\prime} \ldots 0^{\prime}}=\partial_{A}$. The $2 n+2$ vector fields

$$
\nabla_{A A_{1}^{\prime} \ldots A_{n}^{\prime}}=\left\{\partial_{A}, \nabla_{A 0^{\prime} 1^{\prime} A_{2}^{\prime} \ldots A_{n-1}^{\prime}}, D_{A n}\right\}
$$

$\operatorname{span} T^{*} \mathcal{N}$

\subsection{The twistor space for the hierarchy}

The twistor space $\mathcal{P} \mathcal{T}$ for a solution to the hierarchy associated to the Lax system on $\mathcal{N}$ as above is obtained by factoring the spin bundle $\mathcal{N} \times \mathbb{C P}^{1}$ by the twistor distribution (Lax system) $L_{A i}$. This clearly has a projection $q: \mathcal{N} \times \mathbb{C P}^{1} \mapsto \mathcal{P} \mathcal{T}$ and we have a double fibration

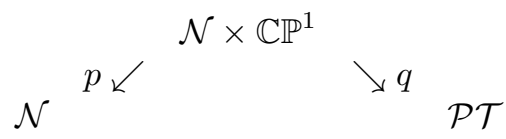

Since the twistor distribution is tangent to the fibres of $\mathcal{N} \times \mathbb{C P}^{1} \mapsto \mathbb{C P}^{1}$, twistor space inherits the projection $\mu: \mathcal{P} \mathcal{T} \mapsto \mathbb{C P}^{1}$. The twistor space for the hierarchy is three-dimensional as for the ordinary hyper-Kähler equations, but has a different topology. We have 
Lemma 4.2 The holomorphic curves $q\left(\mathbb{C P}_{x}^{1}\right)$ where $\mathbb{C P}_{x}^{1}=p^{-1} x, x \in \mathcal{N}$, have normal bundle $N=$ $\mathcal{O}(n) \oplus \mathcal{O}(n)$.

Proof. To see this, note that $N$ can be identified with the quotient $p^{*}\left(T_{x} \mathcal{N}\right) /\left\{\operatorname{span} L_{A i}\right\}, i=1, \ldots, n$. In their homogeneous form the operators $L_{A i}$ have weight 1 , so the distribution spanned by them is isomorphic to the bundle $\mathbb{C}^{2 n} \otimes \mathcal{O}(-1)$. The definition of the normal bundle as a quotient gives

$$
0 \mapsto \mathbb{C}^{2 n} \otimes \mathcal{O}(-1) \mapsto \mathbb{C}^{2 n+2} \mapsto N \mapsto 0
$$

and we see, by taking determinants that the image is $\mathcal{O}(n+a) \oplus \mathcal{O}(n-a)$ for some $a$. We see that $a=0$ as the last map, in the spinor notation introduced at the end of the last section, is given explicitly by $V^{A A_{1}^{\prime} \ldots A_{n}^{\prime}} \mapsto V^{A A_{1}^{\prime} \ldots A_{n}^{\prime}} \pi_{A_{1}^{\prime}} \ldots \pi_{A_{n}^{\prime}}$ clearly projecting onto $\mathcal{O}(n) \oplus \mathcal{O}(n)$.

A final structure that $\mathcal{P} \mathcal{T}$ possesses is a skew form $\Sigma$ taking values in $\mathcal{O}(2 n)$ on the fibres of the projection $\mu$. This arises from the fact that the vector fields of the distribution preserve the coordinate volume form $\nu$ on $\mathcal{N}$ in the given coordinates system. Furthermore, the Lax system commutes exactly $\left[L_{a I}, L_{B j}\right]=0$ so that

$$
\Sigma=\nu\left(\cdot, \cdot, L_{01}, \ldots, L_{0 n}, L_{11}, \ldots, L_{1 n}\right)
$$

descends to the fibres of $\mathcal{P} \mathcal{T} \mapsto \mathbb{C P}^{1}$ and clearly has weight $2 n$ as each of the $L_{A i}$ has weight one.

Thus we see that, given a solution to the hyperkähler hierarchy in the form of a commuting Lax system, we can produce a twistor space with the above structures. Now we shall prove the main result of this section and demonstrate that, given $\mathcal{P} \mathcal{T}$, with the above structures, we can construct $\mathcal{N}$ (as the moduli space of rational curves in $\mathcal{P} \mathcal{T}$ ) which is naturally equipped with a function $\Theta$ satisfying $(4.49)$ and with the Lax distribution (4.50).

Proposition 4.3 Let $\mathcal{P} \mathcal{T}$ be a 3 dimensional complex manifold with the following structures

1) a projection $\mu: \mathcal{P} \mathcal{T} \longrightarrow \mathbb{C P}^{1}$,

2) a section $s: \mathbb{C P}^{1} \mapsto \mathcal{P} \mathcal{T}$ of $\mu$ with normal bundle $\mathcal{O}(n) \oplus \mathcal{O}(n)$,

3) a non-degenerate 2-form $\Sigma$ on the fibres of $\mu$, with values in the pullback from $\mathbb{C P}^{1}$ of $\mathcal{O}(2 n)$.

Let $\mathcal{N}$ be the moduli space of sections that are deformations of the section s given in (2). Then $\mathcal{N}$ is $2 n+2$ dimensional and

a) There exists coordinates, $x^{A i}, A=0,1$, and $i=0, \ldots, n$ and a function $\Theta: \mathcal{N} \longrightarrow \mathbb{C}$ on $\mathcal{N}$ such that equation (4.49) is satisfied.

b) The moduli space $\mathcal{N}$ of sections is equipped with

- a factorisation of the tangent bundle $T \mathcal{N}=S^{A} \otimes \odot{ }^{n} S^{A^{\prime}}$,

- a $2 n$-dimensional distribution on the 'spin bundle' $D \subset T\left(\mathcal{N} \times \mathbb{C P}^{1}\right)$ that is tangent to the fibres of $\mathbf{r}$ over $\mathbb{C P}^{1}$ and, as a bundle on $\mathcal{N} \times \mathbb{C P}^{1}$ has an identification with $\mathcal{O}(-1) \otimes S_{A A^{\prime} \ldots A_{n-1}^{\prime}}$ so that the linear system can be written as in equation (4.50).

This correspondence is stable under small perturbations of the complex structure on $\mathcal{P} \mathcal{T}$ preserving (1) and (3).

Proof: The first claim, that $\mathcal{N}$ has dimension $2 n+2$ follows from Kodaira theory as $\operatorname{dim} H^{0}\left(\mathbb{C P}^{1}, N\right)=$ $2 n+2$ and $\operatorname{dim} H^{1}\left(\mathbb{C P}^{1}, N\right)=\operatorname{dim} H^{1}\left(\mathbb{C P}^{1}, \operatorname{End} N\right)=0$.

Proof of (a): we first start by defining homogeneous coordinates on $\mathcal{P} \mathcal{T}$. These are coordinates on $\mathcal{T}$, the total space of the pullback from $\mathbb{C P}^{1}$ of the tautological line bundle $\mathcal{O}(-1)$. Let $\pi_{A^{\prime}}$ be homogeneous coordinates on $\mathbb{C P}^{1}$ pulled back to $\mathcal{T}$ and let $\omega^{A}$ be local coordinates on $\mathcal{T}$ chosen on a neighbourhood of $\mu^{-1}\left\{\pi_{0^{\prime}}=0\right\}$ that are homogeneous of degree $n$ and canonical so that $\Sigma=\varepsilon_{A B} \mathrm{~d} \omega^{A} \wedge \mathrm{d} \omega^{B}$. We also use $\lambda=\pi_{0^{\prime}} / \pi_{1^{\prime}}$ as an affine coordinate on $\mathbb{C P}^{1}$. Let $L_{p}$ be the line in $\mathcal{P} \mathcal{T}$ that corresponds to $p \in \mathcal{N}$ and let $Z \in \mathcal{P} \mathcal{T}$ lie on $L_{p}$. We denote by $\mathcal{F}$ the correspondence space $\mathcal{P} \mathcal{T} \times\left.\mathcal{N}\right|_{Z \in L_{p}}=\mathcal{N} \times \mathbb{C P}^{1}$. (See figure 1 for the double fibration picture.)

Pull back the twistor coordinates to $\mathcal{F}$ and define $2(n+1)$ coordinates on $\mathcal{N}$ by

$$
x^{A\left(A_{1}^{\prime} A_{2}^{\prime} \ldots A_{n}^{\prime}\right)}:=\left.\frac{\partial^{n} \omega^{A}}{\partial \pi_{A_{1}^{\prime}} \partial \pi_{A_{2}^{\prime}} \ldots \partial \pi_{A_{n}^{\prime}}}\right|_{\pi_{A^{\prime}}=o_{A^{\prime}}},
$$


Figure 1: Double fibration.

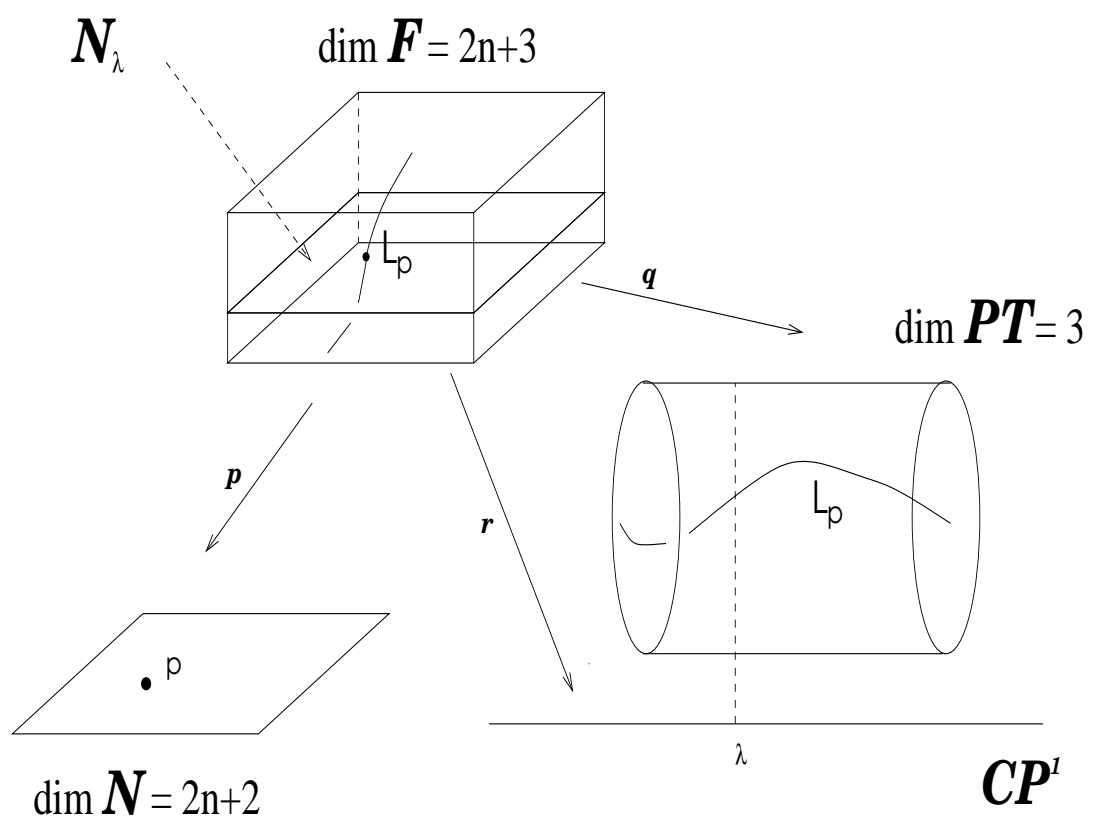

where the derivative is along the fibres of $\mathcal{F}$ over $\mathcal{N}$. This can alternatively be expressed in affine coordinates on $\mathbb{C P}^{1}$ by expanding the coordinates $\omega^{A}$ pulled back to $\mathcal{F}$ in powers of $\lambda=\pi_{0^{\prime}} / \pi_{1^{\prime}}$ :

$$
\omega^{A}=\left(\pi_{1^{\prime}}\right)^{n}\left(\sum_{i=0}^{n} x^{A i} \lambda^{n-i}+\lambda^{n+1} \sum_{i=0}^{\infty} s_{i}^{A} \lambda^{i}\right),
$$

where the $s_{i}^{A}$ are functions of $x^{A A_{1}^{\prime} \ldots A_{n}^{\prime}}$ and will be useful later.

The symplectic 2 -form $\Sigma$ on the fibres of $\mu$, when pulled back to the spin bundle, has expansion in powers of $\lambda$ that truncates at order $2 n+1$ by globality and homogeneity, so that

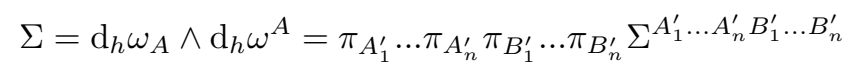

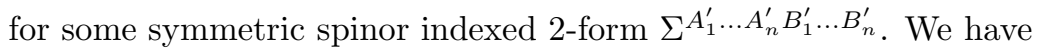

$$
\Sigma(\lambda) \wedge \Sigma(\lambda)=0, \quad \mathrm{~d}_{h} \Sigma(\lambda)=0 .
$$

where in the exterior derivative $\mathrm{d}_{h}, \lambda$ is understood to be held constant.

If we express the forms in terms of the $x^{A i}$ and the $s_{i}^{A}$, the closure condition is satisfied identically, whereas the truncation condition will give rise to equations on the $s_{i}^{A}$ allowing one to express them in terms of a function $\Theta\left(x^{A A^{\prime} \ldots A_{n}^{\prime}}\right)$ and to field equations on $\Theta$ as follows.

To deduce the existence of $\Theta\left(x^{A A_{1}^{\prime} \ldots A_{n}^{\prime}}\right)$ observe that the vanishing of the coefficient of $\lambda^{2 n+1}$ in $\mathrm{d} \omega^{A} \wedge \mathrm{d} \omega_{A}$ gives

$$
\sum_{i=0}^{n} \mathrm{~d} s_{A i} \wedge \mathrm{d} x^{A i}=\mathrm{d} \sum_{i=0}^{n} s_{A i} \mathrm{~d} x^{A i}=0 \quad \Longrightarrow \quad s_{A i}=\frac{\partial \Theta}{\partial x^{A i}} .
$$

The equations of the hierarchy arise from the vanishing of the coefficient of $\lambda^{2 n+2}$

$$
\sum_{i=0}^{n} \mathrm{~d} x^{A i} \wedge \mathrm{d} s_{A}^{i+1}+\mathrm{d} s^{A 0} \wedge \mathrm{d} s_{A}^{0}=0 .
$$

This leads to the equations (4.49) on $\Theta$ for $i, j \leq n-1$,

$$
\frac{\partial^{2} \Theta}{\partial x^{A i+1} \partial x^{B j}}-\frac{\partial^{2} \Theta}{\partial x^{A i} \partial x^{B j+1}}+\varepsilon^{C D} \frac{\partial^{2} \Theta}{\partial x^{C 0} \partial x^{A i}} \frac{\partial^{2} \Theta}{\partial x^{D 0} \partial x^{B j}}=0
$$

and further equations that determine $s^{A n+1}$. 
Proof of b). The isomorphism $T \mathcal{N}=S^{A} \otimes \odot{ }^{n} S^{A^{\prime}}$ follows simply from the structure of the normal bundle. From Kodaira theory, since the appropriate obstruction groups vanish, we have

$$
T_{x} \mathcal{N}=\Gamma\left(\mathbb{C P}_{x}^{1}, N_{x}\right)=S_{x}^{A} \otimes \odot{ }^{n} S^{A^{\prime}}
$$

where $N_{x}$ is the normal bundle to the rational curve $\mathbb{C P}_{x}^{1}$ in $\mathcal{P} \mathcal{T}$ corresponding to the point $x \in \mathcal{N}$. The bundle $S^{A}$ on space-time is the Ward transform of $\mathcal{O}(-n) \otimes T_{V} \mathcal{P} \mathcal{T}$ where the subscript $V$ denotes the sub-bundle of the tangent bundle consisting of vectors up the fibres of $\mu$, the projection to $\mathbb{C P}^{1}$, so that $S_{x}^{A}=\Gamma\left(\mathbb{C P}_{x}^{1}, \mathcal{O}(-n) \otimes T_{V} \mathcal{P} \mathcal{T}\right)$. The bundle $S^{A^{\prime}}=\Gamma\left(\mathbb{C P}^{1}, \mathcal{O}(1)\right)$ is canonically trivial.

Let $\nabla_{A A_{1}^{\prime} \cdots A_{n}^{\prime}}=\nabla_{A\left(A_{1}^{\prime} \cdots A_{n}^{\prime}\right)}$ be the indexed vector field that establishes the isomorphism (4.58) and let $e^{A A_{1}^{\prime} \cdots A_{n}^{\prime}}=e^{A\left(A_{1}^{\prime} \cdots A_{n}^{\prime}\right)} \in \Omega^{1} \otimes S^{A} \otimes \odot{ }^{n} S^{A^{\prime}}$ be the dual (inverse) map.

We now wish to derive the form of the linear system, equations $(4.50)$. For each fixed $\pi_{A^{\prime}}=(\lambda, 1) \in$ $\mathbb{C P}^{1}$ we have a copy of a space-time $\mathcal{N}_{\lambda}$. The horizontal (i.e. holding $\lambda$ constant) subspace of $T_{(x, \lambda)}(\mathcal{N} \times$ $\left.\mathbb{C P}^{1}\right)$ is spanned by $\nabla_{A\left(A^{\prime} \ldots A_{n}^{\prime}\right)}$. An element of the normal bundle to the corresponding line $\mathbb{C P}_{x}^{1}$ consists of a a horizontal tangent vector at $(x, \lambda)$ modulo the twistor distribution. Therefore we have the sequence of sheaves over $\mathbb{C P}^{1}$

$$
0 \longrightarrow D_{x} \longrightarrow T_{x} \mathcal{N} \stackrel{e^{A}}{\longrightarrow} S^{A} \otimes \mathcal{O}(n) \longrightarrow 0,
$$

where $D_{x}$ is the twistor distribution at $x$ and the map $T_{x} \mathcal{N} \longrightarrow S^{A} \otimes \mathcal{O}(n)$ is given by the contraction of elements of $T_{x} \mathcal{N}$ with $e^{A}:=e^{A A_{1}^{\prime} \ldots A_{n}^{\prime}} \pi_{A_{1}^{\prime} \ldots \pi_{A_{n}^{\prime}}}$ since $e^{A}$ annihilates all $L_{B i} \mathrm{~S}$ in $D$. Consider the dual sequence tensored with $\mathcal{O}(-1)$ to obtain

$$
0 \longrightarrow \mathcal{O}_{A}(-n-1) \longrightarrow T_{x}^{*} \mathcal{N}(-1) \longrightarrow D_{x}^{*}(-1) \longrightarrow 0 .
$$

From here we would like to extract the Lax distribution

$$
L_{A A_{2}^{\prime} \ldots A_{n}^{\prime}}=\pi^{A_{1}^{\prime}} D_{A A_{1}^{\prime} A_{2}^{\prime} \ldots A_{n}^{\prime}} \in S_{A A_{2}^{\prime} \ldots A_{n}^{\prime}} \otimes \mathcal{O}(1) \otimes D .
$$

This can be achieved by globalising (4.59) in $\pi^{A^{\prime}}$. The corresponding long exact sequence of cohomology groups yields

$$
\begin{aligned}
0 \longrightarrow \Gamma\left(\mathcal{O}_{A}(-n-1)\right) \longrightarrow & \Gamma\left(T^{*} \mathcal{N}(-1)\right) \longrightarrow \Gamma\left(D^{*}(-1)\right) \stackrel{\delta}{\longrightarrow} H^{1}\left(\mathcal{O}_{A}(-n-1)\right) \\
& \longrightarrow H^{1}\left(T^{*} \mathcal{N}(-1)\right) \longrightarrow \ldots
\end{aligned}
$$

which (because $T^{*} \mathcal{N}$ is a trivial bundle so that $\mathcal{O}(-1) \otimes T^{*} \mathcal{N}$ has no sections or cohomology) reduces to

$$
0 \longrightarrow \Gamma\left(D^{*}(-1)\right) \stackrel{\delta}{\longrightarrow} H^{1}\left(\mathcal{O}_{A}(-n-1)\right) \longrightarrow 0 .
$$

From Serre duality we conclude, since $D$ has rank $2 n$, that the connecting map $\delta$ is an isomorphism $\delta: \Gamma\left(D^{*}(-1)\right) \longrightarrow S_{A A_{2}^{\prime} \ldots A_{n}^{\prime}}$. Therefore

$$
\delta \in \Gamma\left(D \otimes \mathcal{O}(1) \otimes S_{A A_{2}^{\prime} \ldots A_{n}^{\prime}}\right)
$$

is a canonically defined object annihilating $\omega^{A}$ given by (4.56).

In index notation we can put

$$
\delta=L_{A A_{2}^{\prime} \ldots A_{n}^{\prime}}=\pi^{A_{1}^{\prime}} D_{A A_{1}^{\prime} A_{2}^{\prime} \ldots A_{n}^{\prime}},
$$

where $L_{A A_{2}^{\prime} \ldots A_{n}^{\prime}}=L_{A\left(A_{2}^{\prime} \ldots A_{n}^{\prime}\right)}$, the second identity follows from the globality of $L_{A A_{2}^{\prime} \ldots A_{n}^{\prime}}$ and the $D_{A A_{1}^{\prime} A_{2}^{\prime} \ldots A_{n}^{\prime}}$ are vector fields on $\mathcal{N}$ lifted to $\mathcal{N} \times \mathbb{C P}^{1}$ using the product structure.

It follows from $L_{A A_{2}^{\prime} \ldots A_{n}^{\prime}} \omega^{B}=0$ that if $\pi^{A^{\prime}}=o^{A^{\prime}}$ then $D_{A 0^{\prime} A_{2}^{\prime} \ldots A_{n}^{\prime}} x^{B n}=0$ so

$$
D_{A 0^{\prime} A_{2}^{\prime} \ldots A_{n}^{\prime}}=A_{A 0^{\prime} A_{2}^{\prime} \ldots A_{n}^{\prime}}^{B B_{n}^{\prime} \ldots} \frac{\partial}{\partial x^{B 0^{\prime} B_{2}^{\prime} \ldots B_{n}^{\prime}}},
$$

for some matrix $A_{A 0_{2}^{\prime} A_{2}^{\prime} \ldots A_{n}^{\prime}}^{B B_{n}^{\prime}}$. This matrix must be invertible by dimension counting. By multiplying $L_{A A_{2}^{\prime} \ldots A_{n}^{\prime}}$ by the inverse of this matrix, we find we can put

$$
A_{A 0^{\prime} A_{2}^{\prime} \ldots A_{n}^{\prime}}^{B B_{n}^{\prime} \ldots B_{n}^{\prime}}=\varepsilon_{A}^{B} \varepsilon_{A_{2}^{\prime} \ldots \varepsilon_{A_{n}^{\prime}}^{B_{n}^{\prime}}}^{B_{n}^{\prime}} .
$$

Therefore we can take $L_{A A_{2}^{\prime} \ldots A_{n}^{\prime}}=\partial_{A 0^{\prime} A_{2}^{\prime} \ldots A_{n}^{\prime}}-\lambda D_{A 1^{\prime} A_{2}^{\prime} \ldots A_{n}^{\prime}}$. Equating the $(n-i+1)$ th and $(n+1)$ th powers of $\lambda$ in $L_{A i} \omega^{B}=0$ to zero yields

$$
D_{A 1^{\prime} A_{2}^{\prime} \ldots A_{n}^{\prime}}=\partial_{A 1^{\prime} A_{2}^{\prime} \ldots A_{n}^{\prime}}+\left[\partial_{A 0^{\prime} A_{2}^{\prime} \ldots A_{n}^{\prime}}, V\right]
$$

where $V=\varepsilon_{A B} \partial \Theta / \partial x_{A 0} \partial / \partial x_{B 0}$. So finally $L_{A A_{2}^{\prime} \ldots A_{n}^{\prime}}$ is of the form $L_{A i}=\partial_{A i}-\lambda\left(\partial_{A i+1}+\left[\partial_{A i}, V\right]\right)$. 


\subsection{Geometric structures}

If one considers $\mathcal{N}=\mathcal{M} \times \mathbb{X}$ as being foliated by four dimensional slices $t^{A i}=$ const then structures (1)-(3) on $\mathcal{P} \mathcal{T}$ can be used to define anti-self-dual vacuum metrics on the leaves of the foliation. Consider $\Theta\left(x^{A A^{\prime}}, \mathbf{t}\right)$ where $\mathbf{t}=\left\{t^{A i}, i=2 \ldots n\right\}$. For each fixed $\mathbf{t}$ the function $\Theta$ satisfies the second heavenly equation. The ASD metric on a corresponding four-dimensional slice $\mathcal{N}_{\mathbf{t}=\mathbf{t}_{\mathbf{0}}}$ is given by

$$
\mathrm{d} s^{2}=2 \varepsilon_{A B} \mathrm{~d} x^{A 1^{\prime}} \mathrm{d} x^{B 0^{\prime}}+2 \frac{\partial^{2} \Theta}{\partial x^{A 0^{\prime}} \partial x^{B 0^{\prime}}} \mathrm{d} x^{A 1^{\prime}} \mathrm{d} x^{B 1^{\prime}} .
$$

This metric can be determined from the structure of the $\mathcal{O}(n) \oplus \mathcal{O}(n)$ twistor space as follows. by

Fix the first $2 n-2$ parameters in the expansion (4.56) so the normal vector $W=W^{A} \partial / \partial \omega^{A}$ is given

$$
W^{A}=\delta \omega^{A}=\lambda^{n-1} W^{A 1^{\prime}}+\lambda^{n} W^{A 0^{\prime}}+\lambda^{n+1} \frac{\partial \delta \Theta}{\partial x_{A}^{0^{\prime}}}+\ldots
$$

where $\delta \Theta=W^{A A^{\prime}} \partial \Theta / \partial x^{A A^{\prime}}$. The metric is

$$
g(U, W)=\varepsilon_{A B} \varepsilon_{A^{\prime} B^{\prime}} U^{A A^{\prime}} W^{B B^{\prime}}
$$

where $\varepsilon_{A^{\prime} B^{\prime}}$ is a fixed element of $\Lambda^{2} S^{A^{\prime}}$ and $\varepsilon_{A B} \in \Lambda^{2} S^{A}$ is determined by $\Sigma$; recall that $S_{x}^{A}=$ $\Gamma\left(L_{x}, \mathcal{O}(-\backslash) \otimes T_{V} \mathcal{P} \mathcal{T}\right)$. Thus if $u^{A}, v^{A} \in S_{x}^{A}$, then define $\varepsilon_{A B} u^{A} v^{B}=\Sigma(u, v)$ where $u, v$ are the corresponding weighted vertical vector fields on $\mathcal{P} \mathcal{T}$.

For $n$ odd $T \mathcal{N}$ is equipped with a metric with holonomy $S L(2, \mathbb{C})$. For $n$ even, $T \mathcal{N}$ is endowed with a skew form. They are both given by

$$
G(U, W)=\varepsilon_{A B} \varepsilon_{A_{1}^{\prime} B_{1}^{\prime}} \ldots \varepsilon_{A_{n}^{\prime} B_{n}^{\prime}} U^{A A_{1}^{\prime} \ldots A_{n}^{\prime}} W^{B B_{1}^{\prime} \ldots B_{n}^{\prime}} .
$$

These are special examples of the paraconformal structures considered by Bailey and Eastwood [2].

\subsection{Holomorphic deformations and $\mathcal{O}(2 n)$ twistor functions}

We wish to consider holomorphic deformations of $\mathcal{P} \mathcal{T}$ that preserve conditions $(1-3)$ of Proposition 4.3 which will therefore correspond to perturbations of the hierarchy.

Let $\tilde{\omega}^{A}=G^{A}\left(\omega^{B}, \pi_{A^{\prime}}, t\right)$ be the standard patching relation for $\mathcal{P} \mathcal{T}$ and let $f^{A} \in S^{A} \otimes H^{1}(\mathcal{P} \mathcal{T}, \mathcal{O}(n))$ give the infinitesimal deformation

$$
\tilde{\omega}^{A}=G^{A}+t f^{A}+O\left(t^{2}\right) .
$$

The globality of the symplectic structure $\mathrm{d} \tilde{\omega}_{A} \wedge \mathrm{d} \tilde{\omega}^{A}=\mathrm{d} \omega_{A} \wedge \mathrm{d} \omega^{A}$ implies $f^{A}=\varepsilon^{A B} \partial f / \partial \omega^{B}$ where $f \in H^{1}(\mathcal{P} \mathcal{T}, \mathcal{O}(2 n))$.

Example: if we deform from the flat model using $f=\left(\pi_{0^{\prime}}\right)^{4 n} / \omega^{0} \omega^{1}$, then the deformation equations

$$
\tilde{\omega}^{0}=\omega^{0}+t \frac{\left(\pi_{0}\right)^{4 n}}{\omega^{0}\left(\omega^{1}\right)^{2}}+O\left(t^{2}\right), \quad \tilde{\omega}^{1}=\omega^{1}-t \frac{\left(\pi_{0}\right)^{4 n}}{\left(\omega^{0}\right)^{2} \omega^{1}}+O\left(t^{2}\right) .
$$

imply that $Q=\omega^{0} \omega^{1}=\tilde{\omega}^{0} \tilde{\omega}^{1}$ is a global twistor function (up to $O\left(t^{2}\right)$ ) which persists to all orders as $\varepsilon^{A B} \partial Q / \partial \omega^{A} \partial f / \partial \omega^{B}=0$. The corresponding deformed paraconformal structure admits a symmetry corresponding to the global vector field $\varepsilon^{A B} \partial Q / \partial \omega^{A} \partial / \partial \omega^{B}$ on $\mathcal{P} \mathcal{T}$.

To see how such 'Hamiltonians' $f$ correspond to variations in the paraconformal structure (or more simply $\Theta$ ), we form an indexed element of $H^{1}\left(\mathcal{P} \mathcal{T}, \mathcal{O}(-1)\right.$ ), and pull it back to $\mathcal{N} \times \mathbb{C P}^{1}$ where it can be split uniquely:

$$
\pi_{A_{2}^{\prime} \ldots \pi_{A_{n}^{\prime}}} \frac{\partial^{3} f^{2 n}}{\partial \omega^{A} \partial \omega^{B} \partial \omega^{C}}=f_{A B C A_{2}^{\prime} \ldots A_{n}^{\prime}}=\tilde{\mathcal{F}}_{A B C A_{2}^{\prime} \ldots A_{n}^{\prime}}-\mathcal{F}_{A B C A_{2}^{\prime} \ldots A_{n}^{\prime}} .
$$

where

$$
\mathcal{F}_{A B C A_{2}^{\prime} \ldots A_{n}^{\prime}}=\frac{1}{2 \pi i} \oint_{\Gamma} \frac{f_{A B C A_{2}^{\prime} \ldots A_{n}^{\prime}}}{\rho_{A^{\prime}} \pi^{A^{\prime}}} \rho \cdot \mathrm{d} \rho .
$$

This gives rise to a global field that is symmetric over its indices:

$$
C_{A B C D A_{2}^{\prime} \ldots A_{n}^{\prime} D_{2}^{\prime} \ldots D_{n}^{\prime}}=L_{D D_{2}^{\prime} \ldots D_{n}^{\prime}} \mathcal{F}_{A B C A_{2}^{\prime} \ldots A_{n}^{\prime}}
$$

which is given also directly by the integral

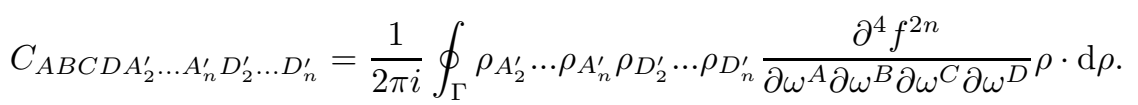


To see how this corresponds to a variation of $\Theta$, we introduce a chain of potentials. Use the non-unique splitting $f^{2 n}=\mathcal{F}^{2 n}-\widetilde{\mathcal{F}}^{2 n}$ and define a global object of degree $2 n+1$ by

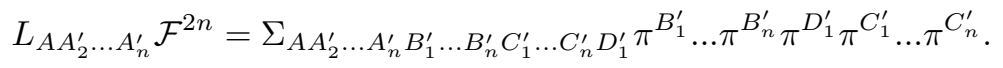

It is easy to see that

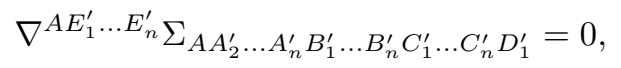

and $\Sigma_{A A_{2}^{\prime} \ldots A_{n}^{\prime} B_{1}^{\prime} \ldots B_{n}^{\prime} C_{1}^{\prime} \ldots C_{n}^{\prime} D_{1}^{\prime}}$ is a potential potentials, related to the field by

$$
C_{A B C D A_{2}^{\prime} \ldots A_{n}^{\prime} D_{2}^{\prime} \ldots D_{n}^{\prime}}=\nabla_{D D_{2}^{\prime} \ldots D_{n}^{\prime}}^{D_{1}^{\prime}} \nabla_{C}^{C_{1}^{\prime} \ldots C_{n}^{\prime}} \nabla_{B}^{B_{1}^{\prime} \ldots B_{n}^{\prime}} \Sigma_{A A_{2}^{\prime} \ldots A_{n}^{\prime} B_{1}^{\prime} \ldots B_{n}^{\prime} C_{1}^{\prime} \ldots C_{n}^{\prime} D_{1}^{\prime} .}
$$

The chain of potentials is

$$
\begin{aligned}
& \delta \Theta_{A_{1}^{\prime} B_{1}^{\prime} \ldots B_{n}^{\prime} C_{1}^{\prime} \ldots C_{n}^{\prime} D_{1}^{\prime}}=o_{A_{1}^{\prime}} O_{B_{1}^{\prime}} \ldots o_{B_{n}^{\prime}} o_{C_{1}^{\prime}} \ldots o_{C_{n}^{\prime}} o_{D_{1}^{\prime}} \delta \Theta
\end{aligned}
$$

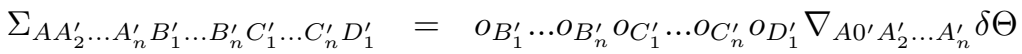

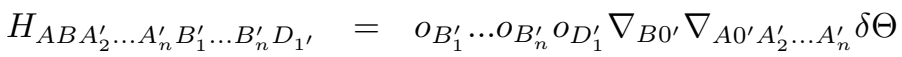

$$
\begin{aligned}
& \Gamma_{A B C A_{2}^{\prime} \ldots A_{n}^{\prime} D_{1}^{\prime}}=o_{D_{1}^{\prime}} \nabla_{C 0^{\prime}} \nabla_{B 0^{\prime}} \nabla_{A 0^{\prime} A_{2}^{\prime} \ldots A_{n}^{\prime}} \delta \Theta \\
& C_{A B C D A_{2}^{\prime} \ldots A_{n}^{\prime} D_{2}^{\prime} \ldots D_{n}^{\prime}}=\nabla_{C 0^{\prime}} \nabla_{B 0^{\prime}} \nabla_{A 0^{\prime} A_{2}^{\prime} \ldots A_{n}^{\prime}} \nabla_{D 0^{\prime} D_{2}^{\prime} \ldots D_{n}^{\prime}} \delta \Theta .
\end{aligned}
$$

This can be compared with the corresponding chain for $n=1$ [14.

\section{Hamiltonian and Lagrangian formalisms}

In this Section we shall investigate the Lagrangian and Hamiltonian formulations of the hyper-Kähler equations in their 'heavenly' forms. The symplectic form on the space of solutions to heavenly equations will be derived, and proven to be compatible with a recursion operator.

Both the first and second heavenly equations admit Lagrangian formulations, and these can be used to derive symplectic structures on the solution spaces, which we denote by $\mathcal{S}$. Here, rather than consider the equations as a real system of elliptic or ultra-hyperbolic equations, we complexify and consider the equations locally as evolving initial data from a 3-dimensional hyper-surface and it is this space of initial data that leads to local solutions on a neighbourhood of such a hyper-surface that is denoted by $\mathcal{S}$ and is endowed with a (conserved) symplectic form.

For the first equation we have the Lagrangian density

$$
\mathcal{L}_{\Omega}=\Omega\left(\nu-\frac{1}{3}(\partial \tilde{\partial} \Omega)^{2}\right)=\left(\Omega-\frac{1}{3} \Omega\left\{\Omega_{\tilde{z}}, \Omega_{\tilde{w}}\right\}_{w z}\right) \nu
$$

and for the second equation

$$
\begin{aligned}
\mathcal{L}_{\Theta} & =\left(\frac{2}{3} \Theta\left(\partial_{2} \Theta\right)^{2}-\frac{1}{2}(\partial \Theta) \wedge\left(\partial_{2} \Theta\right)\right) \wedge e^{A 0^{\prime}} \wedge e_{A}^{0^{\prime}} \\
& =\left(\frac{1}{3} \Theta\left\{\Theta_{x}, \Theta_{y}\right\}_{x y}-\frac{1}{2}\left(\Theta_{x} \Theta_{w}+\Theta_{y} \Theta_{z}\right)\right) \nu .
\end{aligned}
$$

Note that $e^{A 0^{\prime}} \wedge e_{A}^{0^{\prime}}$ can be replaced by $\mathrm{d} x \wedge \mathrm{d} y$ in the second Lagrangian as it is multiplied by $\mathrm{d} w \wedge \mathrm{d} z$.

If the field equations are assumed, the variation of these Lagrangians will yield only a boundary term. Starting with the first equation, this defines a potential one-form $P$ on the solution space $\mathcal{S}$ and hence a symplectic structure $\boldsymbol{\Omega}=\mathrm{d} P$ on $\mathcal{S}$. Starting with the second we find a symplectic structure with the same expression on perturbations $\delta \Theta$ as we had for $\delta \Omega$. However, since their relation to perturbations of the hyper-Kähler structure are different, they define different symplectic structures on $\mathcal{S}$. These are related by the recursion operator since we have $R^{2} \delta \Omega=\delta \Theta$ from above. In order to see that these structures yield the usual bi-Hamiltonian framework, we will need to show that these symplectic structures are compatible with the recursion operator in the sense that $\boldsymbol{\Omega}\left(R \phi, \phi^{\prime}\right)=\boldsymbol{\Omega}\left(\phi, R \phi^{\prime}\right)$.

We shall demonstrate this using the first heavenly formulation which is easier as one can use identities from Kähler geometry. (The derivation of the symplectic structure from the second Lagrangian will be done in coordinates, since the useful relation between the Hodge star and the Kähler structure is missing in this case.)

Proposition 5.1 The symplectic form on the space of solutions $\mathcal{S}$ derived from the boundary term in the variational principle for the first Lagrangian is

$$
\boldsymbol{\Omega}\left(\delta_{1} \Omega, \delta_{2} \Omega\right)=\frac{2}{3} \int_{\delta M} \delta_{1} \Omega * \mathrm{~d}\left(\delta_{2} \Omega\right)-\delta_{2} \Omega * \mathrm{~d}\left(\delta_{1} \Omega\right) .
$$


Proof. Varying (5.63) we obtain

$$
\delta L=\delta \Omega\left(\nu-\frac{1}{3}(\partial \tilde{\partial} \Omega)^{2}\right)-\frac{2}{3} \Omega \partial \tilde{\partial} \Omega \wedge \partial \tilde{\partial} \delta \Omega=\frac{2}{3} \partial \tilde{\partial} \Omega \wedge(\delta \Omega \partial \tilde{\partial} \Omega-\Omega \partial \tilde{\partial} \delta \Omega) .
$$

We use the identities $\mathrm{d}(\partial-\tilde{\partial})=2 \tilde{\partial} \partial, \quad \omega \wedge J_{1} \mathrm{~d}=\partial \tilde{\partial} \Omega \wedge(\partial-\tilde{\partial})=* \mathrm{~d}$ and the field equation to obtain

$$
\begin{aligned}
\delta L & =-\frac{1}{3} \partial \tilde{\partial} \Omega \wedge(\delta \Omega \mathrm{d}(\partial-\tilde{\partial}) \Omega-\Omega \mathrm{d}(\partial-\tilde{\partial}) \delta \Omega) \\
& =\frac{1}{3} \mathrm{~d} A(\delta \Omega)-\frac{1}{3} \partial \tilde{\partial} \Omega(-* \partial \tilde{\partial} \Omega(\partial-\tilde{\partial}) \delta \Omega(\partial-\tilde{\partial}) \Omega+* \partial \tilde{\partial} \Omega(\partial-\tilde{\partial}) \Omega(\partial-\tilde{\partial}) \delta \Omega) \\
& =\frac{1}{3} \mathrm{~d} A(\delta \Omega) \quad \text { where } A(\delta \Omega)=\Omega * \mathrm{~d} \delta \Omega-\delta \Omega * \mathrm{~d} \Omega .
\end{aligned}
$$

Define the one form on $\mathcal{S}$

$$
P=\int_{\delta M} A(\delta \Omega)
$$

The symplectic structure $\boldsymbol{\Omega}$ is the (functional) exterior derivative of $P$

$$
\begin{aligned}
\boldsymbol{\Omega}\left(\delta_{1} \Omega, \delta_{2} \Omega\right) & =\delta_{1}\left(P\left(\delta_{2} \Omega\right)\right)-\delta_{2}\left(P\left(\delta_{1} \Omega\right)\right)-P\left(\left[\delta_{1} \Omega, \delta_{2} \Omega\right]\right) \\
& =\frac{2}{3} \int_{\delta M} \delta_{1} \Omega * \mathrm{~d}\left(\delta_{2} \Omega\right)-\delta_{2} \Omega * \mathrm{~d}\left(\delta_{1} \Omega\right) .
\end{aligned}
$$

Thus $\boldsymbol{\Omega}$ coincides with the symplectic form on the solution space to the wave equation on the ASD vacuum background.

The existence of the recursion operator allows the construction of an infinite sequence of symplectic structures. The key property we need is the following

Proposition 5.2 Let $\phi, \phi^{\prime} \in W_{g}$ and let $\boldsymbol{\Omega}$ be given by (5.65). Then

$$
\boldsymbol{\Omega}\left(R \phi, \phi^{\prime}\right)=\boldsymbol{\Omega}\left(\phi, R \phi^{\prime}\right) .
$$

We first prove a technical lemma:

Lemma 5.3 The following identities hold

$$
\begin{aligned}
\omega \wedge \partial \phi & =-\alpha \wedge \tilde{\partial} R \phi, \quad \omega \wedge \partial_{2} \phi=\tilde{\alpha} \wedge \partial_{2} R \phi, \\
\omega \wedge \partial_{2} R \phi & =-\alpha \wedge \partial_{0} \phi, \quad \omega \wedge \tilde{\partial} R \phi=\tilde{\alpha} \wedge \partial \phi .
\end{aligned}
$$

Proof. From the definitions of $\Sigma^{A^{\prime} B^{\prime}}$ and $\partial_{A^{\prime}}^{B^{\prime}}$ it follows that

$$
\Sigma^{A^{\prime} B^{\prime}} \wedge \partial_{D^{\prime}}^{C^{\prime}}=\Sigma^{A^{\prime}\left[B^{\prime}\right.} \wedge \partial_{D^{\prime}}^{\left.C^{\prime}\right]}
$$

(recall that $\left.\partial_{A^{\prime}}^{B^{\prime}}=e^{A B^{\prime}} \otimes \partial_{A A^{\prime}}\right)$ which yields

$$
\begin{gathered}
\omega \wedge \tilde{\partial}=\tilde{\alpha} \wedge \partial_{2}, \omega \wedge \partial=-\alpha \wedge \partial_{0}, \\
\omega \wedge \partial_{0}=\tilde{\alpha} \wedge \partial, \omega \wedge \partial_{2}=-\alpha \wedge \partial_{2}, \quad \alpha \wedge \partial=\tilde{\alpha} \wedge \tilde{\partial}=0 .
\end{gathered}
$$

Multiplying (3.28) by combinations of spin co-frame we get an equivalent definition of the recursion operator

$$
\partial_{1^{\prime}}^{A^{\prime}} \phi=\partial_{0^{\prime}}^{A^{\prime}} R \phi
$$

which is equivalent to $\partial \phi=\partial_{2} R \phi$ or $\phi=\tilde{\partial} R \phi$. These formulae give the desired result.

Proof of Proposition 5.2. The proof uses a (formal) application of Stokes' theorem:

$$
\begin{aligned}
\boldsymbol{\Omega}\left(\phi, \phi^{\prime}\right) & =\int_{\delta M} \phi * \mathrm{~d} \phi^{\prime}-\phi^{\prime} * \mathrm{~d} \phi \\
& =\int_{\delta M} \omega \wedge\left(\phi \partial \phi^{\prime}-\phi \tilde{\partial} \phi^{\prime}-\phi^{\prime} \partial \phi+\phi^{\prime} \tilde{\partial} \phi\right)=\int_{\delta M} \omega \wedge\left(\phi \mathrm{d} \phi^{\prime}+\phi^{\prime} \mathrm{d} \phi^{\prime}-2 \phi \tilde{\partial} \phi^{\prime}-2 \phi^{\prime} \partial \phi\right) \\
& =-2 \int_{\delta M} \omega \wedge\left(\phi \tilde{\partial} \phi^{\prime}+\phi^{\prime} \partial \phi\right)=2 \int_{\delta M} \omega \wedge\left(\phi^{\prime} \tilde{\partial} \phi+\phi \partial \phi^{\prime}\right) .
\end{aligned}
$$


From (5.70) and from (5.67) we have

$$
\boldsymbol{\Omega}\left(\phi, R \phi^{\prime}\right)=-\int_{\delta M} \omega \wedge\left(\phi \tilde{\partial} R \phi^{\prime}+R \phi^{\prime} \partial \phi\right)=-\int_{\delta M} \phi \partial \phi^{\prime} \wedge \tilde{\alpha}+\int_{\delta M} R \phi^{\prime} \tilde{\partial} R \phi \wedge \alpha
$$

and analogously

$$
\boldsymbol{\Omega}\left(R \phi, \phi^{\prime}\right)=\int_{\delta M} \phi^{\prime} \partial \phi \wedge \tilde{\alpha}-\int_{\delta M} R \phi \tilde{\partial} R \phi^{\prime} \wedge \alpha .
$$

Equality (5.66) is achieved by subtracting the integral of $\mathrm{d}\left(\phi \phi^{\prime}\right) \wedge \tilde{\alpha}-\mathrm{d}\left(R \phi R \phi^{\prime}\right) \wedge \alpha$ and applying Stokes' theorem.

This property guarantees that the bilinear forms

$$
\boldsymbol{\Omega}^{k}\left(\phi, \phi^{\prime}\right) \equiv \boldsymbol{\Omega}\left(R^{k} \phi, \phi^{\prime}\right)
$$

are skew. Furthermore they are symplectic and lead to the bi-Hamiltonian formulation. In this context formula (5.66) and the closure condition for $\Omega^{k}$ are an algebraic consequence of the fact that $R$ comes from two Poisson structures. Using the theory of bi-Hamiltonian systems one can now go on to prove that the flows constructed by application of $R$ to some standard flow commute.

To develop the bi-Hamiltonian theory, we would like to write the heavenly equations in Hamiltonian form. However the Legendre transform becomes singular for the coordinate flows associated to the coordinates we have chosen since they are, at least in the Minkowski space limit, null coordinates. One possibility is to develop a Hamiltonian formalism based on such null hyper-surfaces. We shall adopt a different approach and reformulate the second heavenly equation as a first order system.

Define $\phi:=-\Theta_{x}$ and formally rewrite the second heavenly equation (2.22) as

$$
\partial_{w} \phi=\mathcal{R}\left(\partial_{y} \phi\right) \quad \text { where } \quad \mathcal{R}=\left(\partial_{z}+\{\phi, \ldots\}_{y x}\right) \circ \partial_{x}{ }^{-1}=\nabla_{11^{\prime}} \circ \nabla_{10^{\prime}}{ }^{-1} .
$$

It is therefore a conjugated operator $\mathcal{R}$ (defined by (3.29), acting on solutions to the zero-rest-mass equations, and plays the role of the recursion operator. Flows of the sub-hierarchy $\left[L_{1}, L_{0 j}\right]=0$ are

$$
\partial_{t_{j}} \phi=\mathcal{R}^{j} \partial_{y} \phi
$$

and the Hamiltonian for the first nontrivial flow is

$$
H_{1}=\int \frac{\phi^{2}}{2} \mathrm{~d} x \wedge \mathrm{d} y \wedge \mathrm{d} z .
$$

Higher Hamiltonians $H_{n}$ can in principle be constructed using the operator $R$. However, we have not developed explicit formulae for these $H_{n}$.

\subsection{A local bi-Hamiltonian form for the hierarchy}

To end this section, we express the equations of the second heavenly hierarchy (4.49) in a compact form, and then write it as a (formal) bi-Hamiltonian system on the spin bundle. This will be a rather different framework from that given above in that the Hamiltonian structure will in effect be local to the $x^{A 0}$ plane as opposed to a field theoretic formulation - it is the gravitational analogue of that given for the Bogomolny equations in [19] except that no symmetries are required here (in effect because ASD gravity can be expressed as ASD Yang-Mills with two symmetries but with gauge group the group of area preserving diffeomorphisms). This formulation is therefore presented merely as a curiousity.

Define the $j$ th truncation of $\omega^{A}$ to be

$$
\omega_{j}^{A}=-x^{A 0}+\sum_{m=1}^{j} \lambda^{m} \partial^{A m-1} \Theta,
$$

where $\partial^{A i}=\varepsilon^{A B} \partial / \partial x^{B i}$. (Note that this is truncated at both ends, although the truncation at the lower end and multiplication by a power of $\lambda$ is inessential.)

Lemma 5.4 The truncated heavenly hierarchy is equivalent to

$$
\partial^{B j} \omega^{A}(\lambda)=\left\{\omega^{A}(\lambda), \lambda^{-j} \omega^{B j}(\lambda)\right\}_{y x} .
$$


Proof. First observe that one can sum the Lax system to obtain

$$
\begin{aligned}
-\sum_{i=0}^{j-1} \lambda^{i} L_{A i} & =\lambda^{j} \partial_{A j}+\sum_{i=0}^{j-1} \lambda^{i+1} \varepsilon^{C D} \partial_{C 0} \partial_{A i} \Theta \partial_{D 0}-\partial_{A 0} \\
& =\lambda^{j} \partial_{A j}+\left\{\omega_{A j}, \cdot\right\}_{y x}
\end{aligned}
$$

where $\{f, \cdot\}_{y x}=\varepsilon^{C D} \partial_{C 0} f \partial_{D 0}$.

Thus, since $L_{A i} \omega^{A}=0$, we have

$$
\partial_{B j} \omega^{A}=-\lambda^{-j}\left\{\omega_{j}^{B}, \omega^{A}\right\}
$$

which yields the desired answer.

For the remainder of this section, we shall fix the values of the spinor indices to be $A=0$ and $B=1$. Set

$$
\partial_{j}:=\partial_{1 j}, \Psi:=\omega^{0} \text {, and } \psi_{j}:=\omega_{1 j} .
$$

Equation (5.72) takes the form

$$
\partial_{j} \Psi(\lambda)=\left\{\Psi(\lambda), \lambda^{-j} \psi_{j}(\lambda)\right\}_{y x}
$$

which we rewrite as

$$
\partial_{j} \Psi=\mathcal{D} \frac{\delta h_{j}}{\delta \Psi} .
$$

Here $\mathcal{D}:=\{\Psi(\lambda), \ldots\}_{y x}=\sum_{i=0}^{\infty} \mathcal{D}_{m} \lambda^{m}$ is $\lambda$-dependent Poisson structure, $\mathcal{D}_{0}=\partial_{x}$ and $\mathcal{D}_{m}=\left[\partial_{m-1}, V\right]=$ $D_{0 m}-\partial_{0 m}$ for $m>0$.

The Hamiltonians are

$$
h_{j}(\lambda)=\lambda^{-j} \psi_{j}(\lambda) \Psi(\lambda) .
$$

\section{Outlook - examples with higher symmetries}

This section motivates the study of solutions to heavenly equations which are invariant under some hidden symmetries, e.g. along the higher flows. More generally, one can consider solutions to the hyper-Kähler equations without symmetries, but whose hierarchies do admit symmetries.

In a subsequent paper we shall give a general construction of such metrics based on a generalisation of 30]. We consider the case in which the twistor spaces have a globally defined twistor function homogeneous of degree $n+1$. This implies that the metric admits a Killing spinor (some solutions with this property are given by [7]). Global sections $Q \in H^{0}\left(\mathbb{C P}^{1}, \mathcal{O}(n+1)\right)$ on non-deformed twistor space $\mu: \mathcal{P} \mathcal{T} \longrightarrow \mathbb{C P}^{1}$ will be classified and $Q$-preserving deformations of the complex structure of a neighbourhood of an $\mathcal{O}(1) \oplus \mathcal{O}(1)$ section of $\mu$ will be studied. The cohomology classes determining the deformation will depend on the fibre coordinates of $\mu$ only via $Q$. The canonical forms of patching functions can be derived to give explicit solutions to anti-self-dual ASD vacuum Einstein equation.

There are also further details of the bi-Hamiltonian structure that could usefully be clarified.

\section{Acknowledgments}

We are grateful to Roger Penrose, George Sparling, Paul Tod, Nick Woodhouse, and others for some helpful discussions. Some parts of this work were finished during the workshop Spaces of geodesics and complex methods in general relativity and geometry held in the summer of 1999 at the Erwin Schrödinger Institute in Vienna. We wish to thank ESI for the hospitality and for financial assistance. LJM was supported by NATO grant CRG 950300 .

\section{References}

[1] Ablowitz, M.J. \& Clarkson, P.A. (1992) Solitons, Nonlinear evolution equations and inverse scattering, L.M.S. Lecture note series, 149, CUP.

[2] Bailey, T.N. \& Eastwood, M.G. (1991) Complex paraconformal manifolds - their differential geometry and twistor theory. Forum Math., 3, no. 1, 61-103. 
[3] Boyer, C.P. \& Plebański, J.F.(1977) Heavens and their integral manifolds, J. Math. Phys. 18, 1022-1031.

[4] Boyer, C.P. \& Plebański, J.F.(1985) An infinite hierarchy of conservation laws and nonlinear superposition principles for self-dual Einstein spaces, J. Math. Phys. 26, 229-234.

[5] Boyer, C. \& Winternitz, P. (1989), Symmetries of the self-dual Einstein equations. I. The infinitedimensional symmetry group and its low-dimensional subgroups. J. Math. Phys. 30, 1081-1094.

[6] Dunajski, M. (1998) The Nonlinear Graviton Construction as an Integrable System, DPhil thesis, Oxford University.

[7] Dunajski, M. (1999) The Twisted Photon Associated to Hyper-hermitian Four Manifolds, J. Geom. Phys. 30 266-281.

[8] Dunajski, M. \& Mason, L.J. (1996) Heavenly Hierarchies and Curved Twistor Spaces, Twistor Newsletter 41.

[9] Dunajski, M. \& Mason, L.J. (1997) Integrable flows on moduli of rational curves with normal bundle $\mathcal{O}^{A}(n)$, Twistor Newsletter 42.

[10] Dunajski, M. \& Mason, L.J. (1997) A Recursion Operator for ASD Vacuums and Z.R.M Fields on ASD Backgrounds, Twistor Newsletter 43.

[11] Dunajski, M. Mason, L.J. \& Woodhouse, N.M.J. (1998) From 2D Integrable Systems to Self-Dual Gravity, J. Phys. A: Math. Gen 31, 6019.

[12] Gindikin, S. (1986) On one construction of hyperKähler metrics, Funct. Anal. Appl 20, 82-132.

[13] Grant, J.D.E. (1993) On Self-Dual Gravity, Phys. Rev. D48, 2606-2612.

[14] Ko, B., Ludvigsen, M., Newman, E.T. \& Tod, K.P. (1981) The theory of $\mathcal{H}$ space, Phys. Rep. 71 51-139.

[15] Kodaira, K. (1963) On stability of compact submanifolds of complex manifolds, Am. J. Math. 85, 79-94.

[16] Magri, F. (1978) A simple model of the integrable Hamiltonian equation, J. Math. Phys., 19, 1156-1162.

[17] Mason, L.J. (1990) $\mathcal{H}$-space, a universal integrable system?, Twistor Newsletter 30.

[18] Mason, L.J. \& Newman, E.T. (1989) A connection between the Einstein and Yang-Mills equations, Comm. Math. Phys., 121, 659-668.

[19] Mason, L.J. \& Sparling, G.A.J. (1992) Twistor correspondences for the soliton hierarchies, J. Geom. Phys., 8, 243-271.

[20] Mason, L.J. \& Woodhouse, N.M.J. (1996) Integrability, Self-Duality, and Twistor Theory, L.M.S. Monographs New Series, 15, OUP.

[21] Park, Q.H. (1990) Self-Dual Gravity as a Large-N Limit of the 2D Non-Linear Sigma Model, Phys. Lett., 238A, 287-290.

[22] Penrose, R. (1965) Zero rest-mass fields including gravitation: asymptotic behaviour, Proc. Roy. Soc. London A284, 159-203

[23] Penrose, R. (1976) Nonlinear gravitons and curved twistor theory, Gen. Rel. Grav. 7, 31-52.

[24] Penrose, R. \& Rindler, W. (1986) Spinors and Space-Time, Vol 1, 2, CUP.

[25] Plebański, J.F. (1975) Some solutions of complex Einstein Equations, J. Math. Phys. 16 23952402.

[26] Strachan, I.A.B. (1995) The Symmetry Structure of the Anti-Self-Dual Einstein Hierarchy, J. Math. Phys. 36, 3566-3573.

[27] Sparling, G. A. \& Tod, K.P. (1981) An example of an $\mathcal{H}$-space, J. Math. Phys. 22 331-332.

[28] Takasaki, K. (1989) An infinite number of hidden variables in hyper-Kähler metrics, J. Math. Phys. 30 1515-1521.

[29] Takasaki, K. (1990) Symmetries of hyper-Kähler (or Poisson gauge field ) hierarchy, J. Math. Phys. 31 1877-1888.

[30] Tod, K.P. \& Ward, R.S. (1979) Self-dual metrics with self-dual Killing vectors Proc. R. Soc. A368 411-427.

[31] Ward, R.S. (1977) On self-dual gauge fields, Phys. Lett. 61A, 81-82.

[32] Ward, R.S. (1985) Integrable and solvable systems and relations among them, Phil. Trans. R. Soc. A 315, 451-457. 